\title{
A Thioether-Ligated Cupric Superoxide Model with Hydrogen Atom Abstraction Reactivity
}

Mayukh Bhadra, ${ }^{\ddagger}$ Wesley J. Transue, ${ }^{\psi}$ Hyeongtaek Lim, ${ }^{\psi}$ Ryan E. Cowley, ${ }^{\psi}$ Jung Yoon C. Lee, ${ }^{\ddagger}$ Maxime A. Siegler, ${ }^{\ddagger}$ Patrick Josephs, ${ }^{\lambda}$ Gerald Henkel, ${ }^{\lambda}$ Markus Lerch,${ }^{\varepsilon}$ Siegfried Schindler, ${ }^{\varepsilon}$ Adam Neuba, ${ }^{\lambda}$ Keith O. Hodgson, ${ }^{\psi, \S}$ Britt Hedman, ${ }^{\S}$ Edward I. Solomon ${ }^{\psi, \S}, *$ and Kenneth D. Karlin ${ }^{\ddagger} *$

\#pepartment of Chemistry, The Johns Hopkins University, Baltimore, Maryland 21218, United States

${ }^{\psi}$ Department of Chemistry, Stanford University, Stanford, California 94305, United States

${ }^{\lambda}$ Department of Chemistry, University of Paderborn, Paderborn D-33098, Germany

${ }^{\varepsilon}$ Institute of Inorganic and Analytical Chemistry, Justus-Liebig University of Gießen, Giessen D-35392, Germany

${ }^{\S}$ Stanford Synchrotron Radiation Lightsource, SLAC National Accelerator Laboratory, Stanford University, Menlo Park, California 94025, United States

\section{Contents :}

1. Materials, Methods and Instrumentation S2-S3

2. Single Crystal X-Ray Crystallography S3-S5

3. Oxygenation of $\mathrm{LCu}(\mathrm{I}) \mathrm{B}\left(\mathrm{C}_{6} \mathrm{~F}_{5}\right)_{4}$ Complex S5-S6

4. Thermodynamics of Dioxygen Binding S7-S9

5. Cyclic Voltammetry (CV) Data S9-S10

6. Resonance Raman Spectroscopy S10-S11

7. X-Ray Absorption Spectroscopy S11-S15

8. Density Functional Theory Computations S16-S23

9. Reactivity and Kinetic Studies S23-S30

$\begin{array}{ll}\text { 10. Product Analyses } & \text { S30-S33 }\end{array}$

11. References $\quad$ S34-S35 


\section{Materials, Methods and Instrumentation}

All starting materials used were commercially available analytical grade from Sigma-Aldrich and TCI chemicals. Inhibitor-free 2-methyltetrahydrofuran (2-MeTHF) was purchased from Sigma-Aldrich, distilled under argon from sodium/benzophenone, and sparged with argon prior to use. Acetonitrile was stored under $\mathrm{N}_{2}$ and purified via passage through $2 \mathrm{~cm} \times 60 \mathrm{~cm}$ columns of activated alumina (Innovative Technologies Inc.). Pentane was freshly distilled from calcium hydride under an inert atmosphere and degassed prior to use. Acetone was distilled over regular Drierite ( $\left.>99.5 \% \mathrm{CaSO}_{4}\right)$ under Ar and deoxygenated with Ar before use. Freshly distilled and Arsparged Acetonitrile and THF were stored in the glovebox over $3 \AA$ activated molecular sieves and 2-MeTHF over $4 \AA$ activated molecular sieves for at least 2 days prior to use. $\left[\mathrm{Cu}^{\mathrm{I}}\left(\mathrm{CH}_{3} \mathrm{CN}\right)_{4}\right] \mathrm{B}\left(\mathrm{C}_{6} \mathrm{~F}_{5}\right)_{4},{ }^{1}{ }^{\mathrm{TMG}} \mathrm{TM}_{3}$ tren, ${ }^{2,3-5}\left[\left({ }^{\mathrm{TMG} 3 t r e n} \mathrm{~N}_{4}\right) \mathrm{Cu}\right] \mathrm{B}\left(\mathrm{C}_{6} \mathrm{~F}_{5}\right)_{4} \quad\left([2] \mathrm{B}\left(\mathrm{C}_{6} \mathrm{~F}_{5}\right)_{4}\right),{ }^{6}$ and TMG$\mathrm{N}_{3} \mathrm{~S}^{7}$, and TEMPO-H/D ${ }^{8-9}$ were synthesized according to literature protocols, and their purities were verified by elemental analysis and/or ${ }^{1} \mathrm{H}$ NMR. For cyclic voltammetry, tetra- $n$ butylammonium hexafluorophosphate $\left(\mathrm{TBAPF}_{6}\right)$ was recrystallized from ethanol before use. Synthesis and manipulations of copper salts were performed according to standard Schlenk techniques or in a VAC (Vacuum Atmospheres Company) glovebox (with $\mathrm{O}_{2}$ and $\mathrm{H}_{2} \mathrm{O}$ levels below $1 \mathrm{ppm})$.

Bench-top low-temperature UV-visible experiments were carried out on a Cary Bio-50 spectrophotometer equipped with a liquid nitrogen chilled Unisoku USP-203-A cryostat using a 1 cm modified Schlenk cuvette. NMR spectroscopy was performed on Bruker 300 and $400 \mathrm{MHz}$ instruments with spectra calibrated to either internal tetramethylsilane (TMS) standard or to a residual protio solvent. ${ }^{10}$ EPR measurements were performed on an X-Band Bruker EMX CW EPR controlled with a Bruker ER 041 XG microwave bridge operating at X-band ( $\sim 9 \mathrm{GHz})$ in 5 
mm quartz EPR tubes cooled to $77 \mathrm{~K}$ in a finger Dewar (Wilmad). Single crystal X-ray Diffraction was performed on suitable crystals, which were mounted either on the tip of a glass fiber or on a loop with a tiny amount of Paratone-N oil and transferred to a $\mathrm{N}_{2}$ cold stream (110(2) K).

$\left[\left({ }^{\mathrm{TMG}} \mathrm{N}_{3} \mathrm{~S}\right) \mathrm{Cu}\right] \mathrm{B}\left(\mathrm{C}_{6} \mathrm{~F}_{5}\right)_{4}\left([1] \mathrm{B}\left(\mathrm{C}_{6} \mathrm{~F}_{5}\right)_{4}\right)$ was synthesized as follows: In a $250 \mathrm{ml}$ Schlenk flask in the glove box, $157.9 \mathrm{mg}(\sim 0.41 \mathrm{mmol})$ of ${ }^{\mathrm{TMG}} \mathrm{N}_{3} \mathrm{~S}$ ligand $(\mathrm{mol} \mathrm{wt}=387.63 \mathrm{grams} / \mathrm{mol})$ were dissolved in $7 \mathrm{~mL}$ of 2-MeTHF. 0.9 equivalents of $\left[\mathrm{Cu}^{\mathrm{I}}\left(\mathrm{CH}_{3} \mathrm{CN}\right)_{4}\right] \mathrm{B}\left(\mathrm{C}_{6} \mathrm{~F}_{5}\right)_{4}(336.2 \mathrm{mg}$, mol $\mathrm{wt}=906.79 \mathrm{grams} / \mathrm{mol}$ ) was added to the solution slowly, yielding a dark yellow solution which was allowed to stir for $5 \mathrm{~min}$ after which approximately $100 \mathrm{~mL}$ of degassed pentane were added to the copper(I) solution. The bilayered mixture was allowed to sit undisturbed overnight and the solvents were allowed to mix. The supernatant was then decanted and the pale-yellow oil was removed from the glove box and dried under vacuum for $10 \mathrm{~min}$ affording a semi-crystalline bright yellow powder which was further dissolved again in 2-MeTHF and recrystallization from pentane resulted in bright yellow crystals of diffraction quality (used for X-Ray crystallography) (87\% yield). Elemental analysis: Calcd for $\mathrm{C}_{42} \mathrm{H}_{41} \mathrm{BCuF}_{20} \mathrm{~N}_{7} \mathrm{~S}$ : $\mathbf{C}, 44.63 ; \mathbf{H}, 3.66 ; \mathbf{N}, 8.68$. Found: $\mathbf{C}$, $44.60 ; \mathbf{H}, 3.64 ; \mathbf{N}, 7.95$.

\section{Single Crystal $X-R a y$ Crystallography of $[1] B\left(C_{6} F_{5}\right)_{4}$}

All reflection intensities were measured at 110(2) K using a SuperNova diffractometer (equipped with Atlas detector) with $\mathrm{Cu} K \alpha$ radiation $(\lambda=1.54178 \AA$ ) under the program CrysAlisPro (Version 1.171.36.32 Agilent Technologies, 2013). The same program was used to refine the cell dimensions and for data reduction. The structure was solved with the program SHELXS-2014/7 (Sheldrick, 2015) and was refined on $F^{2}$ with SHELXL-2014/7 (Sheldrick, 2015). Analytical numeric absorption correction using a multifaceted crystal model was applied using CrysAlisPro. The temperature of the data collection was controlled using the system Cryojet (manufactured by Oxford Instruments). The H atoms were placed at 
calculated positions using the instructions AFIX 23 or AFIX 137 with isotropic displacement parameters having values 1.2 or 1.5 Ueq of the attached $\mathrm{C}$ atoms. The structure is ordered.

Table S1. Experimental details

\begin{tabular}{|c|c|}
\hline & {$[1] \mathrm{B}\left(\mathrm{C}_{6} \mathrm{~F}_{5}\right)_{4}$} \\
\hline \multicolumn{2}{|l|}{ Crystal data } \\
\hline Chemical formula & $\mathrm{C}_{24} \mathrm{BF}_{20} \cdot \mathrm{C}_{18} \mathrm{H}_{41} \mathrm{CuN}_{7} \mathrm{~S}$ \\
\hline$M_{\mathrm{r}}$ & 1130.23 \\
\hline $\begin{array}{l}\text { Crystal system, space } \\
\text { group }\end{array}$ & Triclinic, $P-1$ \\
\hline Temperature (K) & 110 \\
\hline$a, b, c(\AA)$ & $13.7877(3), 13.9488(4), 25.8489(5)$ \\
\hline$\alpha, \beta, \gamma\left(^{\circ}\right)$ & $79.8825(19), 81.9382(18), 74.458(2)$ \\
\hline$V\left(\AA^{3}\right)$ & $4692.2(2)$ \\
\hline$Z$ & 4 \\
\hline Radiation type & $\mathrm{Cu} \mathrm{K \alpha}$ \\
\hline$\mu\left(\mathrm{mm}^{-1}\right)$ & 2.17 \\
\hline Crystal size $(\mathrm{mm})$ & $0.50 \times 0.12 \times 0.05$ \\
\hline \multicolumn{2}{|l|}{ Data collection } \\
\hline Diffractometer & SuperNova, Dual, $\mathrm{Cu}$ at zero, Atlas \\
\hline Absorption correction & $\begin{array}{l}\text { Analytical } \\
\text { CrysAlis PRO, Agilent Technologies, Version 1.171.36.32 (release 02-08- } \\
2013 \text { CrysAlis171 .NET) (compiled Aug } 2 \text { 2013,16:46:58) Analytical } \\
\text { numeric absorption correction using a multifaceted crystal } \\
\quad \text { model based on expressions derived by R.C. Clark \& J.S. Reid. } \\
\text { (Clark, R. C. \& Reid, J. S. (1995). Acta Cryst. A51, 887-897) }\end{array}$ \\
\hline$T_{\min }, T_{\max }$ & $0.579,0.902$ \\
\hline $\begin{array}{l}\text { No. of measured, } \\
\text { independent } r \text { and } \\
\text { observed }[I>2 \sigma(I)] \\
\text { reflections }\end{array}$ & $59936,18322,16042$ \\
\hline$R_{\text {int }}$ & 0.040 \\
\hline$(\sin \theta / \lambda)_{\max }\left(\AA^{-1}\right)$ & 0.616 \\
\hline \multicolumn{2}{|l|}{ Refinement } \\
\hline $\begin{array}{l}R\left[F^{2}>2 \sigma\left(F^{2}\right)\right], w R\left(F^{2}\right), \\
S\end{array}$ & $0.038,0.105,1.03$ \\
\hline No. of reflections & 18322 \\
\hline
\end{tabular}




\begin{tabular}{|l|l|}
\hline No. of parameters & 1315 \\
\hline H-atom treatment & H-atom parameters constrained \\
\hline$\Delta \rho_{\max }, \Delta \rho_{\min }\left(\mathrm{e} \AA^{-3}\right)$ & $0.55,-0.56$ \\
\hline
\end{tabular}

Computer programs: CrysAlis PRO, Agilent Technologies, Version 1.171.36.32 (release 02-08-2013 CrysAlis171 .NET) (compiled Aug 2 2013, 16:46:58), SHELXS2014/7 (Sheldrick, 2015), SHELXL2014/7 (Sheldrick, 2015), SHELXTL v6.10 (Sheldrick, 2008)

Reference: Sheldrick, G. M. (2015). Acta Cryst. C71, 3-8.

\section{Oxygenation of $[1] B\left(C_{6} F_{5}\right)_{4}$ to form $\left[1 \cdot O_{2}\right] B\left(C_{6} F_{5}\right)_{4}$}

In a general procedure, a stock solution of $2.0 \mathrm{mM}[1] \mathrm{B}\left(\mathrm{C}_{6} \mathrm{~F}_{5}\right)_{4}$ was made by dissolving the solid complex in 2-MeTHF in a scintillation vial in the glovebox. This was further diluted to $0.4 \mathrm{mM}$ and $2.5 \mathrm{ml}$ of this solution was transferred in a Schlenk cuvette via syringe, capped with septum, secured with parafilm, and taken out of the glovebox. The cuvette was allowed to cool to $-135{ }^{\circ} \mathrm{C}$ in the cryostat of the UV-Vis spectrophotometer for $15 \mathrm{~min}$ and then dioxygen was gently bubbled through a metallic needle. Dioxygen was bubbled over 5-6 minutes in intervals of $30 \mathrm{~s}$, giving full conversion to $\left[\mathbf{1} \cdot \mathrm{O}_{2}\right] \mathrm{B}\left(\mathrm{C}_{6} \mathrm{~F}_{5}\right)_{4}$. At this point, the spectrum was recorded (Figure $\mathbf{S 1}$ ). The solution containing $\left[\mathbf{1} \cdot \mathrm{O}_{2}\right]^{+}$was subjected to vacuum-argon cycles and Ar-bubbling to remove excess $\mathrm{O}_{2}$ and subsequently warmed up to room temperature following which the cuvette was cooled back to $-135{ }^{\circ} \mathrm{C}$ and the spectrum was recorded while cooling. Figure S2 depicts the reversible regeneration of $\left[\mathbf{1} \cdot \mathrm{O}_{2}\right]^{+}$. The blue spectrum (Figure S1) corresponds to the warmed-up RT product which reversibly binds the released $\mathrm{O}_{2}$ from the cuvette-headspace over a period of $\sim 10$ minutes upon gradual cooling of the solution back to $-135{ }^{\circ} \mathrm{C}$. Comparison of the $442 \mathrm{~nm}$ absorbance (green spectrum of Figures $\mathbf{S 1}$ and $\mathbf{S 2}$ ) indicates $\sim 95 \%$ regeneration of $\left[\mathbf{1} \cdot \mathrm{O}_{2}\right]^{+}$. 


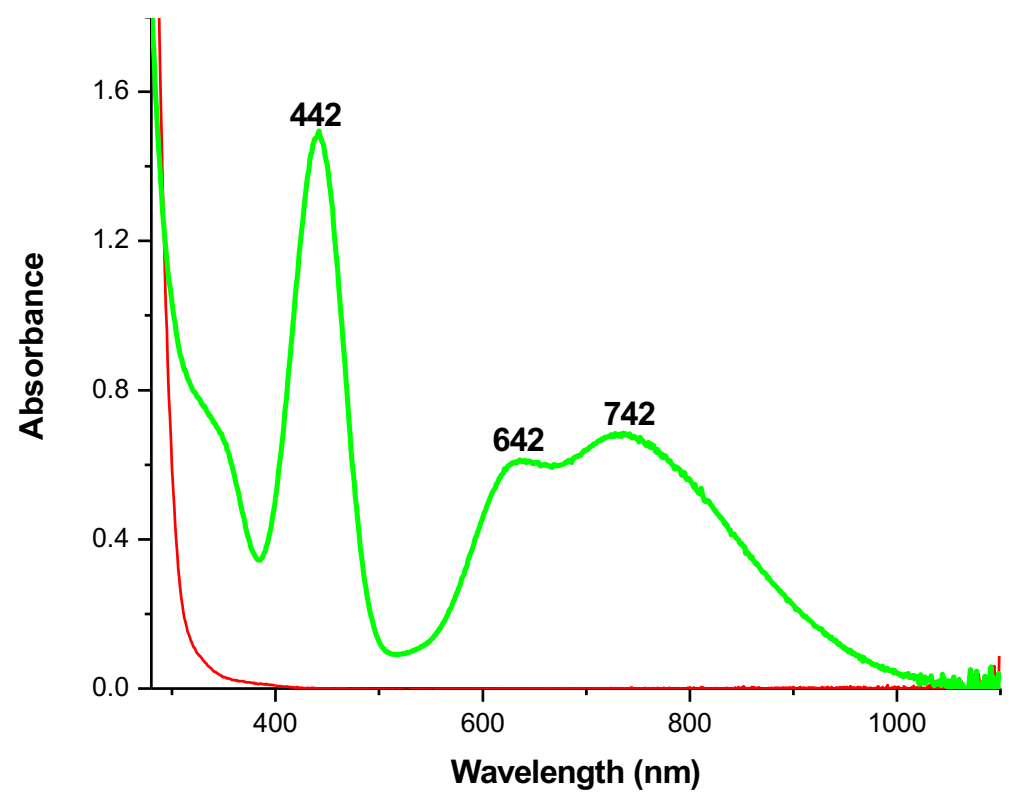

Figure S1. Generation of $\left[1 \cdot \mathrm{O}_{2}\right] \mathrm{B}\left(\mathrm{C}_{6} \mathrm{~F}_{5}\right)_{4}$ features (green spectrum) from the $\mathrm{Cu}^{\mathrm{I}}$ precursor (red spectrum), $0.4 \mathrm{mM}$ solution of $[1] \mathrm{B}\left(\mathrm{C}_{6} \mathrm{~F}_{5}\right)$, upon dioxygen addition at $-135^{\circ} \mathrm{C}$.

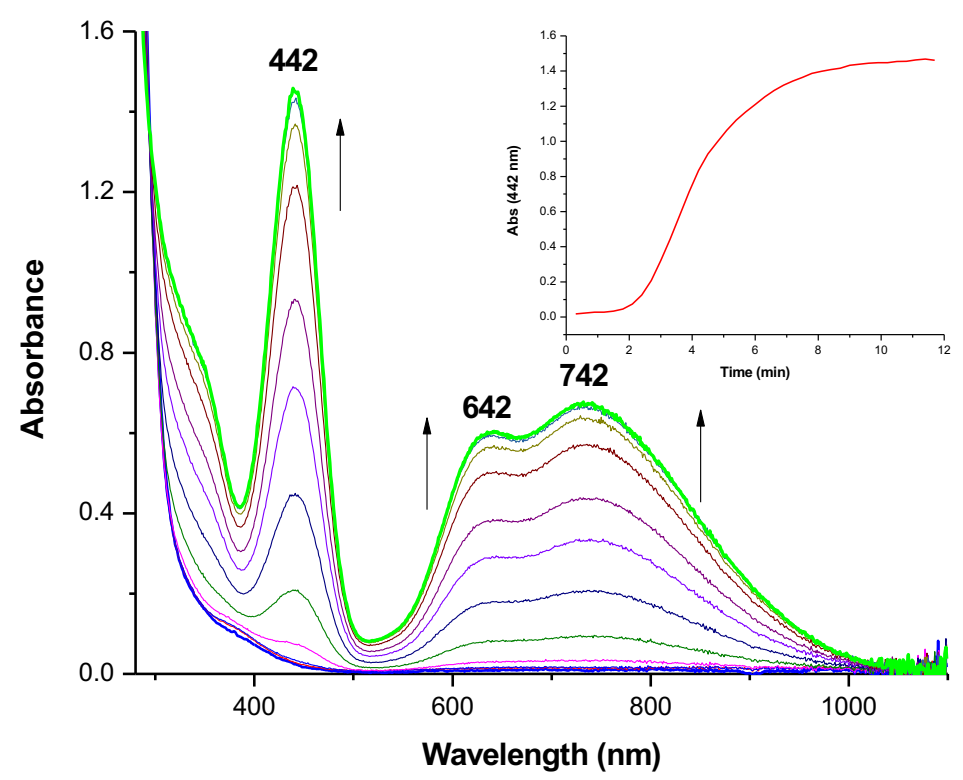

Figure S2. Reversible regeneration of $\left[\mathbf{1}^{\cdot} \mathrm{O}_{2}\right] \mathrm{B}\left(\mathrm{C}_{6} \mathrm{~F}_{5}\right)_{4}$ features (green spectrum) from the warmed up solution at $\mathrm{RT}$ (blue spectrum), upon re-cooling back to $-135{ }^{\circ} \mathrm{C}$; (inset) time trace of the growth of the $442 \mathrm{~nm}$ band of $\left[1 \cdot \mathrm{O}_{2}\right] \mathrm{B}\left(\mathrm{C}_{6} \mathrm{~F}_{5}\right)_{4}$, as recorded while gradual cooling of the experimental setup to $-135^{\circ} \mathrm{C}$. 


\section{Thermodynamics of Dioxygen Binding}

In a cuvette, a $2.5 \mathrm{ml} 2-\mathrm{MeTHF}$ solution of $[1] \mathrm{B}\left(\mathrm{C}_{6} \mathrm{~F}_{5}\right)_{4}$ was taken and excess dioxygen gas was bubbled through a metallic needle in the pre-chilled cuvette in the cryostat. After the full formation of $\left[\mathbf{1}^{\cdot} \mathrm{O}_{2}\right]^{+}$, the headspace and the solution were purged with alternating vacuum and argon cycles. Starting from the lowest temperature, the cuvette was slowly warmed up at increments of $5-10{ }^{\circ} \mathrm{C}$, the headspace $\mathrm{O}_{2}$ was carefully equilibrated to $1 \mathrm{~atm}$ pressure via a mineral oil bubbler and the solution was kept at each temperature for $\sim 15 \mathrm{~min}$, and then the spectra are recorded. Figure 3a in the main manuscript summarizes the spectral change corresponding to the decay of $\left[\mathbf{1}^{\cdot} \mathrm{O}_{2}\right]^{+}$over a range of -145 to $-85^{\circ} \mathrm{C}$.

The temperature-dependent absorbance at $740 \mathrm{~nm}$ is plotted in Figure $\mathbf{3 b}$ (orange plot) in the main manuscript. Slowly warming a solution of $\left[\mathbf{1}^{\cdot} \mathrm{O}_{2}\right]^{+}$saturated with $\mathrm{O}_{2}\left(-145\right.$ to $\left.-85{ }^{\circ} \mathrm{C}\right)$ allowed quantification of $\left[\mathbf{1} \cdot \mathrm{O}_{2}\right]^{+}$at several temperatures. At $-125{ }^{\circ} \mathrm{C}$, ca. $50 \%$ remained and no $\left[\mathbf{1}^{\cdot} \mathrm{O}_{2}\right]^{+}$ was observed above $-90{ }^{\circ} \mathrm{C}$. Analogous measurements on $\left[2 \cdot \mathrm{O}_{2}\right] \mathrm{B}\left(\mathrm{C}_{6} \mathrm{~F}_{5}\right)_{4}\left(-85\right.$ to $\left.+15{ }^{\circ} \mathrm{C}\right)$ generated from $[2] \mathrm{B}\left(\mathrm{C}_{6} \mathrm{~F}_{5}\right)_{4}$ were carried out and the spectral changes are shown in Figure $\mathbf{S 3}$ below, revealing the inflection point of the curve to be ca. $-40{ }^{\circ} \mathrm{C}$ (Figure $3 \mathbf{b}$ (blue plot) in the main text). 


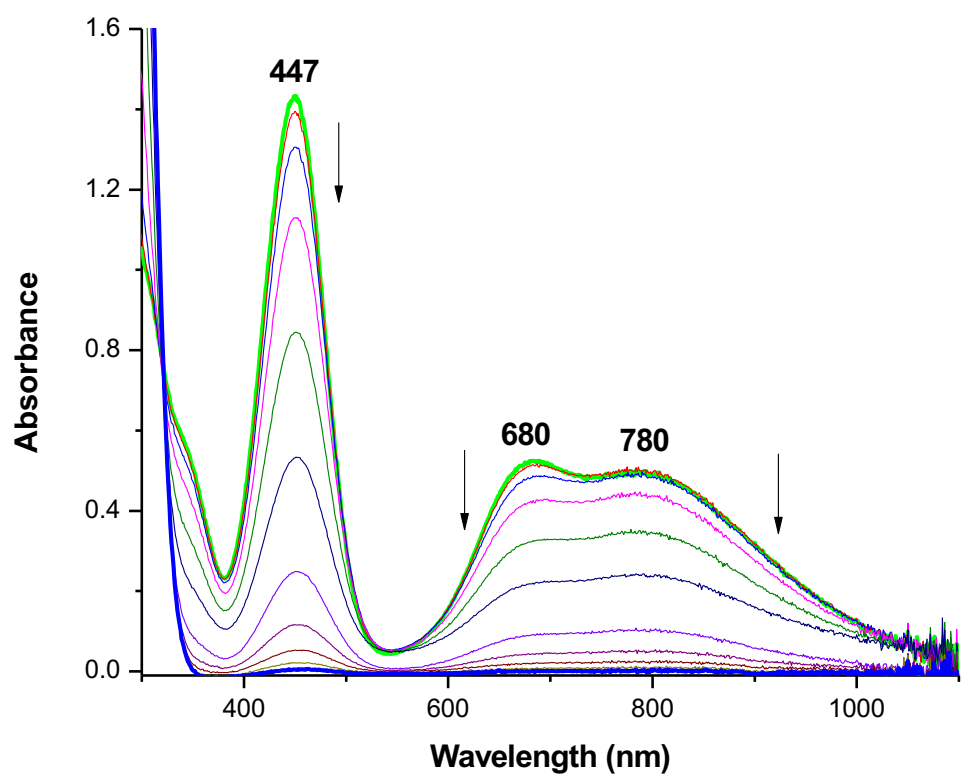

Figure S3. Decay of the $\left[2 \cdot \mathrm{O}_{2}\right]^{+}$features upon incremental warming of the superoxide solution from $-85{ }^{\circ} \mathrm{C}$ (green spectrum) to $+15^{\circ} \mathrm{C}$ (blue spectrum). The temperature-dependent absorbance at $740 \mathrm{~nm}$ is plotted in Figure $\mathbf{3 b}$ (blue plot) in the main text.

The relative thermodynamics of dioxygen binding by $[1] \mathrm{B}\left(\mathrm{C}_{6} \mathrm{~F}_{5}\right)_{4}$ and $[2] \mathrm{B}\left(\mathrm{C}_{6} \mathrm{~F}_{5}\right)_{4}$ were quantified by fitting the variable temperature UV-Vis data at $740 \mathrm{~nm}$ for superoxide formation. This wavelength was selected because it is positioned centrally within the ligand field transitions of both $\left[1 \cdot \mathrm{O}_{2}\right]^{+}$and $\left[2 \cdot \mathrm{O}_{2}\right]^{+}$. Additionally, it avoids the $\sim 680 \mathrm{~nm}$ region, as the $680 \mathrm{~nm}$ band of $\left[2 \cdot \mathrm{O}_{2}\right]^{+}$appears to narrow slightly at the lowest temperatures measured. Such a lineshape change would skew single-wavelength fits. Similar fits to those at $740 \mathrm{~nm}$ are obtained at 447 and $780 \mathrm{~nm}$ wavelengths.

The relation between equilibrium constant $K_{e q}=\frac{\left[\mathrm{CuO}_{2}\right]}{\left[{\mathrm{Cu}]\left[\mathrm{O}_{2}\right]}^{2}\right.}$ and free energy is $\Delta G=\Delta H-T \Delta S=$ $-R T \ln K_{e q}$, where $\left[\mathrm{CuO}_{2}\right]$ is the concentration of the superoxide complex $\left[\mathbf{1} \cdot \mathrm{O}_{2}\right]^{+}$or $\left[\mathbf{2} \cdot \mathrm{O}_{2}\right]^{+},[\mathrm{Cu}]$ the concentration of the cuprous complex $[1]^{+}$or $[2]^{+},\left[\mathrm{O}_{2}\right]$ the concentration of dissolved oxygen, $\Delta H$ the enthalpy change, $\Delta S$ the entropy change, $R$ the gas constant, and $T$ the temperature. 
Application of balance allows conversion into $\left[\mathrm{CuO}_{2}\right]=\frac{[\mathrm{Cu}]_{\text {initial }}}{1+\exp \left(\frac{\Delta H-T \Delta S}{R T}\right) /\left[\mathrm{O}_{2}\right]}$, where $[\mathrm{Cu}]_{\text {initial }}$ is the initial concentration of $[1]^{+}$or $[2]^{+}$before $\mathrm{O}_{2}$ introduction. Accurate $\mathrm{O}_{2}$ solubility data for 2MeTHF are lacking, so we instead assume it follows Henry's law, giving $\left[\mathrm{O}_{2}\right]=$ $\mathcal{H}_{0} \exp \left[-\frac{\Delta H_{\text {sol }}}{R}\left(\frac{1}{T}-\frac{1}{T_{0}}\right)\right]$ for a reference solubility $\mathcal{H}_{0}$ at some reference temperature $T_{0}$ and enthalpy of dissolution $\Delta H_{\text {sol. }}$ Combination of everything with Beer's law gives the superoxide absorption to be $A_{\mathrm{CuO} 2}=\frac{\alpha}{1+\exp (\beta+\gamma / T)}$, where $\alpha=\epsilon l\left[\mathrm{CuO}_{2}\right]$ for extinction coefficient $\varepsilon(740 \mathrm{~nm})$ and cuvette path length $l, \beta=-\ln \mathcal{H}_{0}-\frac{\Delta S}{R}-\frac{\Delta H_{\text {sol }}}{R T_{0}}$, and $\gamma=-\frac{\Delta H_{\text {sol }}}{R}$. By comparing fitted values of composite variables " $\beta$ " and " $\gamma$ ", we obtain relative enthalpic $(\Delta \Delta H=+3.2 \mathrm{kcal} / \mathrm{mol})$ and entropic $(\Delta \Delta S=+3.7 \mathrm{cal} / \mathrm{mol} \mathrm{K})$ thermodynamics. The fits are shown in Figure $\mathbf{3 b}$ of the main manuscript.

\section{Cyclic Voltammetry (CV) Data}

All cyclic voltammograms were collected under an inert argon atmosphere in dry and degassed 17:3 2-MeTHF:Acetone at $25^{\circ} \mathrm{C}$ (Note: The acetone was necessary to dissolve $\mathrm{TBAPF}_{6}$, which did not dissolve in neat 2-MeTHF). The concentration of the cuprous complexes $\left([\mathbf{1}]^{+}\right.$or $\left.[2]^{+}\right)$was $\sim 1.0 \mathrm{mM}$, with $\mathrm{TBAPF}_{6}$ used as the supporting electrolyte ( $0.1 \mathrm{M}$ concentration). A three-electrode system was used as follows: $\mathrm{Ag} / \mathrm{AgNO}_{3}(10 \mathrm{mM})$ in $\mathrm{CH}_{3} \mathrm{CN}$ as reference electrode; glassy carbon electrode as the working electrode; platinum wire as the counter electrode. All reduction potentials were referenced $v s . \mathrm{Fc}^{+/ 0}$ as an external standard under the same conditions. The data obtained are shown in Figure 3c in the main manuscript. 
A similar analysis was performed by dissolving the complexes $\left([1]^{+}\right.$or $\left.[2]^{+}\right)$in the "morecoordinating" $\mathrm{CH}_{3} \mathrm{CN}$ solvent and the data obtained are shown below in Figure $\mathbf{S 4}$.

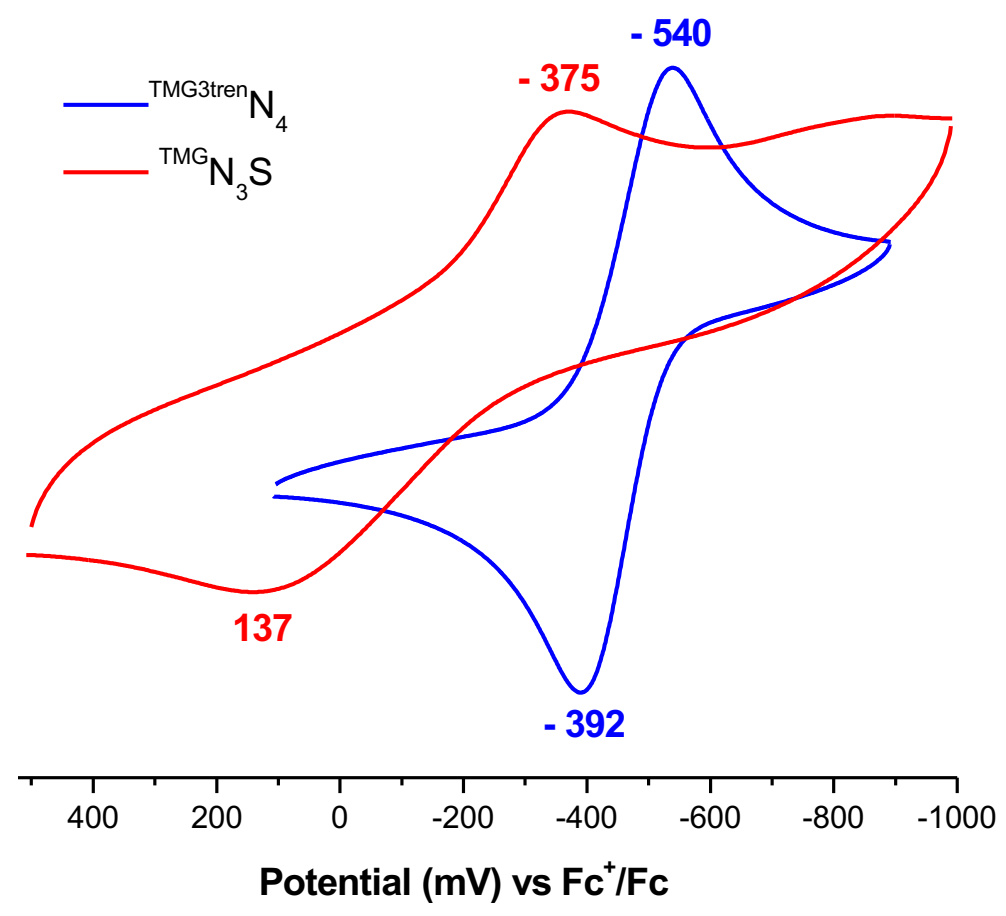

Figure S4. Cyclic voltammograms of the $\left[(\mathrm{L}) \mathrm{Cu}^{\mathrm{I}}\right] \mathrm{B}\left(\mathrm{C}_{6} \mathrm{~F}_{5}\right)_{4}$ complexes in $\mathrm{MeCN}$ at room temperature under $\mathrm{Ar}$ atmosphere. In red is depicted the voltammogram of $\mathrm{Cu}^{\mathrm{II}} / \mathrm{Cu}^{\mathrm{I}}$ couple for $[\mathbf{1}]^{+} /[\mathbf{1}]^{2+}\left(\mathrm{L}=\mathrm{TMG}-\mathrm{N}_{3} \mathrm{~S}\right)$ and in blue that for $[2]^{+} /[2]^{2+}\left(\mathrm{L}=\mathrm{TMG}_{3}\right.$ tren $)$. All reduction potentials were referenced $v s . \mathrm{Fc}^{+/ 0}$ as an external standard under the same conditions as the $\left[(\mathrm{L}) \mathrm{Cu}^{\mathrm{I}}\right] \mathrm{B}\left(\mathrm{C}_{6} \mathrm{~F}_{5}\right)_{4}$ complexes.

\section{Resonance Raman Spectroscopy}

For resonance Raman (rRaman) measurements, a $\sim 1.0 \mathrm{mM}$ stock solution of $[1] \mathrm{B}\left(\mathrm{C}_{6} \mathrm{~F}_{5}\right)_{4}$ was prepared in 2-MeTHF in the glovebox. A $500 \mu \mathrm{L}$ aliquot of this copper(I) solution was added to a $5 \mathrm{~mm}$ NMR sample tube, capped with a septum, and chilled in a 2-MeTHF/ $\mathrm{N}_{2}(l)$ bath. Oxygenation of the copper samples was achieved by slowly bubbling an excess of dioxygen through the solution 
using a Hamilton gas-tight syringe equipped with a three-way valve and needle outlet. Dioxygen, ${ }^{16} \mathrm{O}_{2}$ (Airgas OX UHP-300) or ${ }^{18} \mathrm{O}_{2}$ (Icon 6393), was added to an evacuated Schlenk flask fitted with a septum for the oxygenation reactions described above. rRaman samples were excited at 413.1 $\mathrm{nm}$ using a Coherent $\mathrm{I} 90 \mathrm{C}-\mathrm{K} \mathrm{Kr}^{+}$ion laser while the sample was immersed in a liquid nitrogen-cooled (77 K) EPR finger dewar (Wilmad). Power was measured at the sample as 10 $\mathrm{mW}$. Data were recorded while manually rotating the sample to minimize photodecomposition. The spectra were recorded using a Spex 1877 CP triple monochromator with either a 600, 1200, or 2400 groves/mm holographic spectrograph grating, and detected by an Andor Newton CCD cooled to $-80^{\circ} \mathrm{C}$. Spectra were calibrated on the energy axis to an external sample of toluene at room temperature. Peak positions were determined from fitting the experimental data with Gaussian transitions using Peakfit Version 4. Figure 3d in the main text shows the rRaman data obtained for $\left[\mathbf{1} \cdot \mathrm{O}_{2}\right] \mathrm{B}\left(\mathrm{C}_{6} \mathrm{~F}_{5}\right)_{4}$ with both natural abundance ${ }^{16} \mathrm{O}_{2}$ and ${ }^{18} \mathrm{O}_{2}$.

\section{X-Ray Absorption Spectroscopy}

The $\mathrm{Cu}$ K-edge X-ray absorption spectroscopy (XAS) data were collected at the Stanford Synchrotron Radiation Lightsource (SSRL) on the unfocused 20-pole, $2 \mathrm{~T}$ wiggler side-station beam line 7-3 under storage ring parameters of $3 \mathrm{GeV}$ and $\sim 500 \mathrm{~mA}$. A Si(220) double-crystal monochromator was used for energy selection. The $\mathrm{M}_{0}$ mirror was not employed and the monochromator was detuned by $\sim 50 \%$ to remove contributions from higher harmonics. The 2 MeTHF solutions of $[\mathbf{1}] \mathrm{B}\left(\mathrm{C}_{6} \mathrm{~F}_{5}\right)_{4}$ and $\left[\mathbf{1} \cdot \mathrm{O}_{2}\right] \mathrm{B}\left(\mathrm{C}_{6} \mathrm{~F}_{5}\right)_{4}$ were loaded into delrin XAS cells with 38 $\mu \mathrm{m}$ Kapton windows and stored in liquid $\mathrm{N}_{2}$. During data collection, the samples were maintained at a constant temperature of $\sim 10 \mathrm{~K}$ using an Oxford Instruments CF 1208 liquid helium cryostat. A Canberra 30-element Ge solid-state detector and soller slits equipped with a Ni filter were used 
to collect $\mathrm{Cu} \mathrm{K} \alpha$ fluorescence data. Internal energy calibration was accomplished by simultaneous measurement of the absorption of a $\mathrm{Cu}$ foil placed between two ionization chambers situated after the sample. The first inflection point of the foil spectrum was assigned to $8980.3 \mathrm{eV}$. The extended X-ray absorption fine structure (EXAFS) data are reported to $k=12.8 \AA^{-1}$ to avoid interference from the $\mathrm{Zn} \mathrm{K}$-edge. Over the course of data collection, $[1] \mathrm{B}\left(\mathrm{C}_{6} \mathrm{~F}_{5}\right)_{4}$ did not show photodamage, while photoreduction was observed for $\left[\mathbf{1}^{\cdot} \mathrm{O}_{2}\right] \mathrm{B}\left(\mathrm{C}_{6} \mathrm{~F}_{5}\right)_{4}$. Thus, only the first scans from the fresh sample spots were used for the final average data of $\left[1 \cdot \mathrm{O}_{2}\right] \mathrm{B}\left(\mathrm{C}_{6} \mathrm{~F}_{5}\right)_{4}$. The data presented here include an average of 24 scans and 19 scans for $[1] \mathrm{B}\left(\mathrm{C}_{6} \mathrm{~F}_{5}\right)_{4}$ and $\left[1 \cdot \mathrm{O}_{2}\right] \mathrm{B}\left(\mathrm{C}_{6} \mathrm{~F}_{5}\right)_{4}$, respectively.

Background subtraction and normalization of the data were performed using PySpline. ${ }^{11}$ The data were processed by fitting a second-order polynomial to the pre-edge region and subtracting this from the entire spectrum as a background. A three-region spline of orders 2, 3, and 3 was used to model the smoothly decaying post-edge region. The data were normalized by scaling the spline function to an edge jump of 1.0 at $9000 \mathrm{eV}$. The EXAFS curve-fitting analysis program OPT in EXAFSPAK (George, G. N. Stanford Synchrotron Radiation Laboratory: Stanford, CA, 2000) was used to fit the EXAFS data. The theoretical phase and amplitude functions were generated by FEFF (version 7.0). ${ }^{12}$ Starting structural models were obtained from the DFT optimized structures. During the fitting process, the bond distance $(R)$ and the mean-square thermal and static deviation in $R\left(\sigma^{2}\right)$, which is related to the Debye-Waller factor, were allowed to vary. The threshold energy $\left(E_{0}\right)$, the point at which the photoelectron wave vector $k$ is 0 , was also allowed to vary but was constrained as a common value for all components in a given fit. The amplitude reduction factor $\left(S_{0}^{2}\right)$ was fixed to a value of 1.0 and the coordination number $(\mathrm{CN})$ were systematically varied to achieve the best fit to the EXAFS data and their Fourier transforms. 
(a)

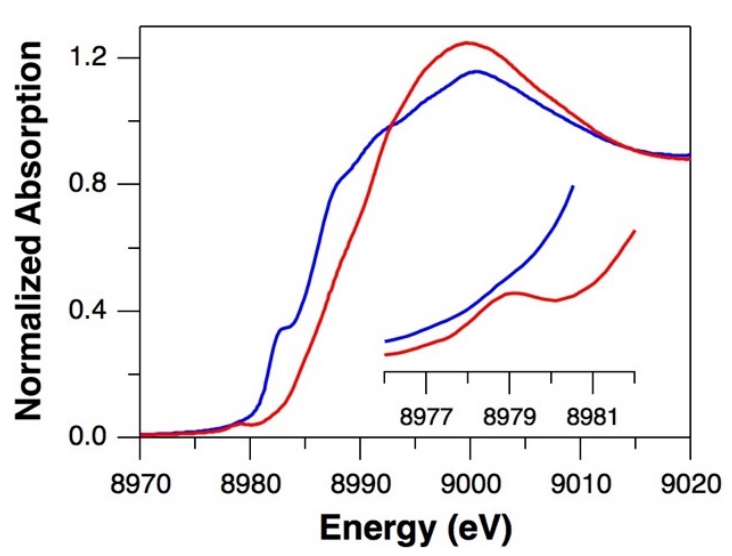

(c)

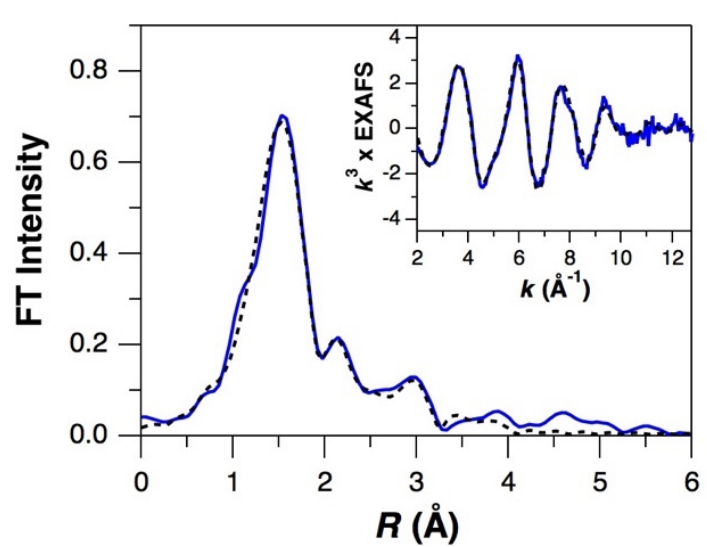

(e)

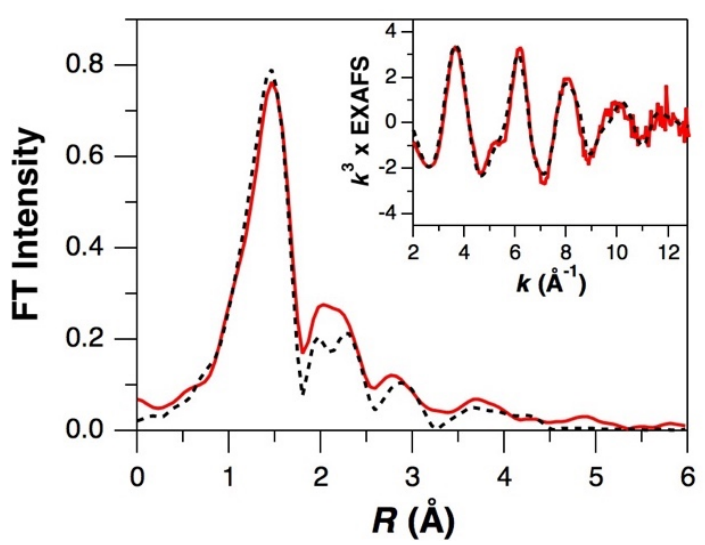

(b)

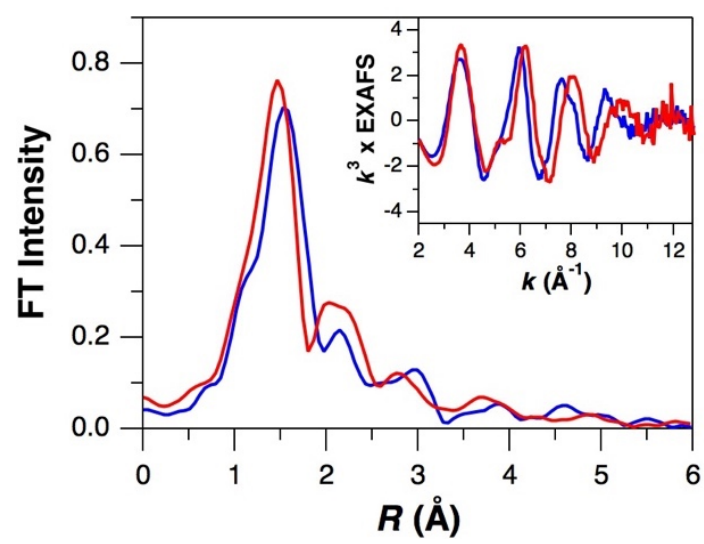

(d)

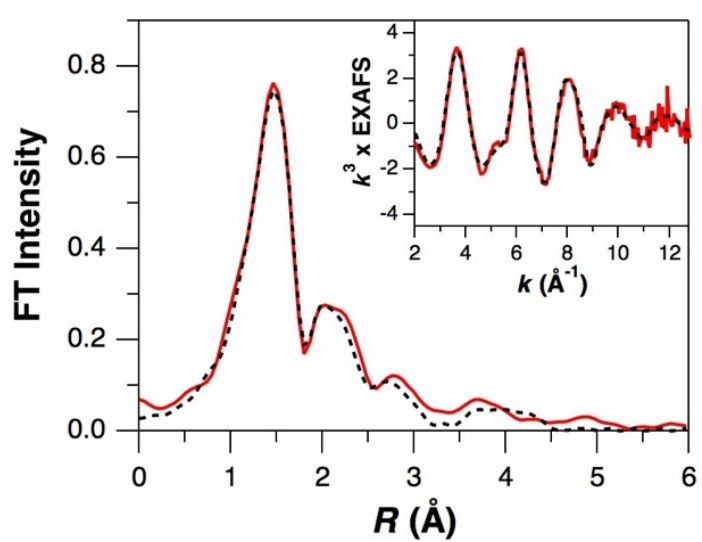

(f)

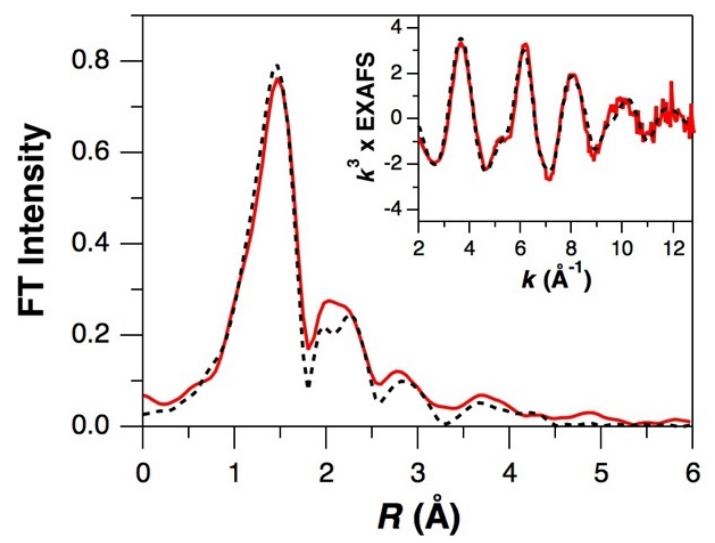

Figure S5. XAS studies on $[1] \mathrm{B}\left(\mathrm{C}_{6} \mathrm{~F}_{5}\right)_{4}$ (blue) and $\left[1 \cdot \mathrm{O}_{2}\right] \mathrm{B}\left(\mathrm{C}_{6} \mathrm{~F}_{5}\right)_{4}$ (red). (a) $\mathrm{Cu} \mathrm{K}$-edge XAS spectra of both compounds with an inset showing the expanded pre-edge region. (b) Comparison of Cu K-edge EXAFS data (inset) and non-phase-shift-corrected Fourier transforms. (c, d) Best fits for EXAFS data (data solid, fit dashed). (e, f) Fits for $\left[\mathbf{1} \cdot \mathrm{O}_{2}\right] \mathrm{B}\left(\mathrm{C}_{6} \mathrm{~F}_{5}\right)_{4}$ replacing the $\mathrm{Cu}-\mathrm{S}$ interaction by (e) $\mathrm{Cu}-\mathrm{C}$ or (f) $\mathrm{Cu}-\mathrm{O}$ (data solid, fit dashed). 
Table S2. Best Fits for the Cu K-edge EXAFS Data

\begin{tabular}{llllll}
\hline Complex & $\mathrm{CN} / \mathrm{Path}^{a}$ & $R(\AA)^{b}$ & $\sigma^{2}\left(\AA^{2}\right)^{c}$ & $\Delta E_{0}(\mathrm{eV})$ & Error $F^{d}$ \\
\hline \hline$[\mathbf{1}] \mathrm{B}\left(\mathrm{C}_{6} \mathrm{~F}_{5}\right)_{4}$ & $3 \mathrm{Cu}-\mathrm{N} / \mathrm{O}$ & 2.01 & 824 & -13.64 & 0.17 \\
& $1 \mathrm{Cu}-\mathrm{S}$ & 2.26 & 651 & & \\
$10 \mathrm{Cu}-\mathrm{N}-\mathrm{C}$ & 3.22 & 822 & & \\
& $4 \mathrm{Cu}-\mathrm{C} / \mathrm{N}$ & 3.45 & 839 & & \\
& $6 \mathrm{Cu}-\mathrm{N} / \mathrm{C}-\mathrm{N} / \mathrm{C}$ & 4.31 & 934 & & \\
& & & & & \\
{$\left[\mathbf{1} \cdot \mathrm{O}_{2}\right] \mathrm{B}\left(\mathrm{C}_{6} \mathrm{~F}_{5}\right)_{4}$} & $4 \mathrm{Cu}-\mathrm{O} / \mathrm{N}$ & 1.98 & 943 & -9.68 & 0.21 \\
& $1 \mathrm{Cu}-\mathrm{S}$ & 2.55 & 1171 & & \\
& $14 \mathrm{Cu}-\mathrm{N}-\mathrm{C}$ & 3.29 & 504 & & \\
& $6 \mathrm{Cu}-\mathrm{N} / \mathrm{C}-\mathrm{N} / \mathrm{C}$ & 4.29 & 927 & & \\
& $16 \mathrm{Cu}-\mathrm{N} / \mathrm{C}-\mathrm{N} / \mathrm{C}$ & 4.66 & 495 & & \\
\hline
\end{tabular}

${ }^{a} \mathrm{CN}$ is coordination number. ${ }^{b}$ The estimated standard deviations in $R$ are $\pm 0.02 \AA$. ${ }^{c}$ The $\sigma^{2}$ values are multiplied by $10^{5}$. ${ }^{d}$ The error $F$ is given by $\left[\sum k^{6}\left(\chi_{\text {exptl }}-\chi_{\text {calcd }}\right)^{2} / \sum k^{6} \chi_{\text {exptl }}\right]^{1 / 2}$. Errors in coordination numbers are $\pm 25 \%$ and those in the identity of the scatterer $Z$ are \pm 1 . 
Table S3. EXAFS Fitting Results for $\left[1 \cdot \mathrm{O}_{2}\right] \mathrm{B}\left(\mathrm{C}_{6} \mathrm{~F}_{5}\right)_{4}$ with $\mathrm{Cu}-\mathrm{C}$ or $\mathrm{Cu}-\mathrm{O}$ Contribution

\begin{tabular}{|c|c|c|c|c|}
\hline $\mathrm{CN} / \mathrm{Path}^{a}$ & $R(\AA)^{b}$ & $\sigma^{2}\left(\AA^{2}\right)^{c}$ & $\Delta E_{0}(\mathrm{eV})$ & Error $F^{d}$ \\
\hline \multicolumn{5}{|l|}{ with $\mathrm{Cu}-\mathrm{C}$} \\
\hline $4 \mathrm{Cu}-\mathrm{O} / \mathrm{N}$ & 1.96 & 948 & -12.24 & 0.25 \\
\hline $1 \mathrm{Cu}-\mathrm{C}$ & 2.85 & 452 & & \\
\hline $14 \mathrm{Cu}-\mathrm{N}-\mathrm{C}$ & 3.29 & 383 & & \\
\hline $6 \mathrm{Cu}-\mathrm{N} / \mathrm{C}-\mathrm{N} / \mathrm{C}$ & 4.24 & 893 & & \\
\hline $12 \mathrm{Cu}-\mathrm{N} / \mathrm{C}-\mathrm{N} / \mathrm{C}$ & 4.63 & 351 & & \\
\hline \multicolumn{5}{|l|}{ with $\mathrm{Cu}-\mathrm{O}$} \\
\hline $4 \mathrm{Cu}-\mathrm{O} / \mathrm{N}$ & 1.96 & 947 & -12.08 & 0.24 \\
\hline $1 \mathrm{Cu}-\mathrm{O}$ & 2.80 & 602 & & \\
\hline $14 \mathrm{Cu}-\mathrm{N}-\mathrm{C}$ & 3.30 & 453 & & \\
\hline $6 \mathrm{Cu}-\mathrm{N} / \mathrm{C}-\mathrm{N} / \mathrm{C}$ & 4.25 & 906 & & \\
\hline $12 \mathrm{Cu}-\mathrm{N} / \mathrm{C}-\mathrm{N} / \mathrm{C}$ & 4.63 & 468 & & \\
\hline
\end{tabular}




\section{Density Functional Theory Computations}

Calculations were performed using ORCA $4.2 .1^{13}$ at the RIJCOSX-B3LYP-D3(BJ)/Def2-TZVP level of theory ${ }^{14-20}$ with a conductor-like polarizable continuum mode ${ }^{21}$ to model tetrahydrofuran solvation. Geometry optimizations were initiated from the crystal structure. The optimized structures largely agreed with experimental results, showing an increase in copper-sulfur distance upon $\mathrm{O}_{2}$ binding (calculated $2.32 \AA$ to $2.50 \AA$; experiment $2.27 \AA$ to $2.55 \AA$ ). Comparison of vibrational levels with experiment was complicated by mixing of modes with the ligand, but a weighted average gave $378 \mathrm{~cm}^{-1}(\mathrm{Cu}-\mathrm{O})$ and $1173 \mathrm{~cm}^{-1}(\mathrm{O}-\mathrm{O})$.

\subsection{Optimized Coordinates of $[1]^{+}$}

$\begin{array}{llll}\mathrm{Cu} & -0.04516643556445 & 0.00032479526531 & -0.00885512610058 \\ \mathrm{~N} & -0.05223785932137 & -0.01782617142287 & -2.27039192911036 \\ \mathrm{C} & -1.44175972981732 & -0.36919125007648 & -2.57312666111229 \\ \mathrm{H} & -1.71759512574404 & -0.07598841920926 & -3.59615796886599 \\ \mathrm{H} & -1.53413865345154 & -1.45331096032946 & -2.50904619457946 \\ \mathrm{C} & -2.42350246998953 & 0.26095303398839 & -1.58713067748671 \\ \mathrm{H} & -3.43404169365740 & -0.06824149083584 & -1.84718112607095 \\ \mathrm{H} & -2.40560969244515 & 1.34681560984768 & -1.71844681141356 \\ \mathrm{~N} & -2.08213901902418 & -0.10898433006512 & -0.21763674566101 \\ \mathrm{C} & -3.02239922462542 & -0.19495211527417 & 0.68004449259237 \\ \mathrm{~N} & -2.82798850098680 & -0.93999276597462 & 1.81400092970899 \\ \mathrm{C} & -3.26279311418169 & -0.45836584167445 & 3.11761949088148 \\ \mathrm{H} & -3.83938681037761 & 0.45519288040610 & 3.01017636908091 \\ \mathrm{H} & -3.87457732300594 & -1.20570422314509 & 3.62843945624212 \\ \mathrm{H} & -2.39126871806135 & -0.24240338453026 & 3.74399941024732 \\ \mathrm{C} & -1.80580495711239 & -1.96513784291048 & 1.85435097471860 \\ \mathrm{H} & -0.82893908125248 & -1.56397884493047 & 2.14880187434787 \\ \mathrm{H} & -2.10163436753502 & -2.72396733922047 & 2.58109521547119 \\ \mathrm{H} & -1.70322225352346 & -2.42385490792588 & 0.87498578710693 \\ \mathrm{~N} & -4.25379044346487 & 0.40642204425437 & 0.56657877299263 \\ \mathrm{C} & -5.47800919340446 & -0.22545571476135 & 1.02832194261291 \\ \mathrm{H} & -5.89343835099879 & 0.27844620555042 & 1.90729892621495\end{array}$




\begin{tabular}{|c|c|c|c|}
\hline $\mathrm{H}$ & -6.22593926623366 & -0.18841441271607 & 0.23143560629757 \\
\hline $\mathrm{H}$ & -5.28687595028228 & -1.26571693820219 & 1.27711307493158 \\
\hline $\mathrm{C}$ & -4.39644447912035 & 1.75907771608174 & 0.05935137413341 \\
\hline $\mathrm{H}$ & -3.42431767901639 & 2.23848799115268 & -0.00683147776184 \\
\hline $\mathrm{H}$ & -4.87202192451293 & 1.78700461763779 & -0.92559825353747 \\
\hline $\mathrm{H}$ & -5.01338853170498 & 2.33711350897725 & 0.75298591383134 \\
\hline $\mathrm{C}$ & 0.30876288655164 & 1.36640937806953 & -2.58869992341292 \\
\hline $\mathrm{H}$ & 0.66796505796465 & 1.46121093506287 & -3.62254147011509 \\
\hline $\mathrm{H}$ & -0.58698581970125 & 1.97890209581045 & -2.49940031310166 \\
\hline $\mathrm{C}$ & 1.36876849778492 & 1.91345361914001 & -1.63247081912706 \\
\hline $\mathrm{H}$ & 1.57404180068273 & 2.95510260508116 & -1.89756766026532 \\
\hline $\mathrm{H}$ & 2.30210039652158 & 1.36481966434874 & -1.79210389042714 \\
\hline $\mathrm{N}$ & 0.91436555819503 & 1.80097445485727 & -0.25020071434891 \\
\hline $\mathrm{C}$ & 1.31351344049602 & 2.66684343655218 & 0.63663459979539 \\
\hline $\mathrm{N}$ & 0.57766254248374 & 2.87593129435609 & 1.77750281756531 \\
\hline $\mathrm{C}$ & 1.23281534414666 & 2.95872806032538 & 3.07642466893744 \\
\hline $\mathrm{H}$ & 2.30376489610357 & 3.08448656502855 & 2.95277645685045 \\
\hline $\mathrm{H}$ & 0.84095852617790 & 3.79807703890558 & 3.65476584901628 \\
\hline $\mathrm{H}$ & 1.05892003331951 & 2.03761839823591 & 3.64399429649317 \\
\hline $\mathrm{C}$ & -0.80950988555837 & 2.46223277519814 & 1.83046002278683 \\
\hline $\mathrm{H}$ & -0.91601495141745 & 1.40405248013358 & 2.09951690379009 \\
\hline $\mathrm{H}$ & -1.32568266566802 & 3.06351062998041 & 2.58093056368088 \\
\hline $\mathrm{H}$ & -1.27600310074691 & 2.61057167184594 & 0.86122664077159 \\
\hline $\mathrm{N}$ & 2.44108087487793 & 3.44167605614597 & 0.50431454951717 \\
\hline $\mathrm{C}$ & 2.50869628701587 & 4.81460828115036 & 0.97493881311676 \\
\hline $\mathrm{H}$ & 3.16693263328798 & 4.91906848377819 & 1.84381816415649 \\
\hline $\mathrm{H}$ & 2.89992231399820 & 5.45066782314878 & 0.17627104126515 \\
\hline $\mathrm{H}$ & 1.51512734995333 & 5.16274038457117 & 1.24365062454426 \\
\hline $\mathrm{C}$ & 3.67753013140690 & 2.89440537736077 & -0.02454861042308 \\
\hline $\mathrm{H}$ & 3.61788212706640 & 1.81053554975758 & -0.06390539025533 \\
\hline $\mathrm{H}$ & 3.90977160706532 & 3.27372457814542 & -1.02417400140174 \\
\hline $\mathrm{H}$ & 4.50019139422221 & 3.16448824465403 & 0.64306138507072 \\
\hline $\mathrm{C}$ & 0.92043323896915 & -1.00403364412846 & -2.73127288127327 \\
\hline $\mathrm{H}$ & 0.69098872257058 & -1.34725932594234 & -3.75020816462292 \\
\hline $\mathrm{H}$ & 1.89823406566090 & -0.52693546401876 & -2.77867246135361 \\
\hline $\mathrm{C}$ & 1.00518912019847 & -2.23221082172950 & -1.82635293054533 \\
\hline $\mathrm{H}$ & 0.06006290030511 & -2.77458254799642 & -1.80680190773266 \\
\hline $\mathrm{H}$ & 1.76610541469768 & -2.91947277553722 & -2.19480524621220 \\
\hline S & 1.33580605955069 & -1.85952335072191 & -0.06521264817578 \\
\hline $\mathrm{C}$ & 3.05393553809058 & -1.23929997078411 & -0.11206523906866 \\
\hline $\mathrm{H}$ & 3.20361149049926 & -0.82182321599591 & 0.88392447141699 \\
\hline $\mathrm{H}$ & 3.10201111239157 & -0.41409251095453 & -0.82057023101982 \\
\hline $\mathrm{C}$ & 4.09277333687476 & -2.30357371967227 & -0.42451949528829 \\
\hline $\mathrm{H}$ & 3.95223784288073 & -2.72147913222490 & -1.42225467908863 \\
\hline $\mathrm{H}$ & 5.09197840918264 & -1.86346354018284 & -0.38674652703178 \\
\hline $\mathrm{H}$ & 4.04907632031361 & -3.11889131171215 & 0.29874669580428 \\
\hline
\end{tabular}




\subsection{Optimized Coordinates of $\left[1 \cdot \mathrm{O}_{2}\right]^{+}$}

\begin{tabular}{|c|c|c|c|}
\hline $\mathrm{Cu}$ & 0.13435586004532 & -0.17339561825732 & -0.04636153348699 \\
\hline $\mathrm{O}$ & 0.20224109574098 & -0.22887774765788 & 1.94536633439382 \\
\hline $\mathrm{O}$ & 1.40223131722906 & -0.25916315008569 & 2.40723583837849 \\
\hline $\mathrm{N}$ & 0.08590102857705 & -0.18668179114921 & -2.24303135388981 \\
\hline $\mathrm{C}$ & -1.31955075006380 & -0.48401282746371 & -2.58979704689674 \\
\hline $\mathrm{H}$ & -1.44094531522387 & -1.56606207085639 & -2.60130166987141 \\
\hline $\mathrm{H}$ & -1.55282710204030 & -0.10846573091327 & -3.59244101453434 \\
\hline $\mathrm{C}$ & -2.27432112405880 & 0.09941504222866 & -1.55871458733715 \\
\hline $\mathrm{H}$ & -2.22946468311746 & 1.19231208545163 & -1.60166545934042 \\
\hline $\mathrm{H}$ & -3.29682370597231 & -0.18598736816010 & -1.81981219075702 \\
\hline $\mathrm{N}$ & -1.89415412444397 & -0.40690576573921 & -0.24639378718262 \\
\hline $\mathrm{C}$ & -2.80378570057920 & -0.62394211807643 & 0.67065188937224 \\
\hline $\mathrm{N}$ & -2.58519390278842 & -1.55374740083098 & 1.64084649938911 \\
\hline $\mathrm{C}$ & -2.96916256810040 & -1.32598649292645 & 3.02502889358641 \\
\hline $\mathrm{H}$ & -2.07562884224263 & -1.34663939271903 & 3.65441613175879 \\
\hline $\mathrm{H}$ & -3.66207473247765 & -2.09426919355340 & 3.37796997128741 \\
\hline $\mathrm{H}$ & -3.43806266387035 & -0.35193051332905 & 3.12941752830696 \\
\hline $\mathrm{C}$ & -1.71673490435906 & -2.69376946963370 & 1.41642546808830 \\
\hline $\mathrm{H}$ & -1.61413375463635 & -2.86834309667185 & 0.34923153455775 \\
\hline $\mathrm{H}$ & -2.16597834344501 & -3.57349117649111 & 1.88361219450867 \\
\hline $\mathrm{H}$ & -0.72477623599889 & -2.53979732015059 & 1.84532443129069 \\
\hline $\mathrm{N}$ & -4.00933604407209 & 0.02165887805609 & 0.70870194198104 \\
\hline $\mathrm{C}$ & -5.23382925424169 & -0.63176029455850 & 1.14368485167284 \\
\hline $\mathrm{H}$ & -5.07061595150445 & -1.70168011831058 & 1.23587010636451 \\
\hline $\mathrm{H}$ & -6.01430260025072 & -0.45918602303386 & 0.39836899452336 \\
\hline $\mathrm{H}$ & -5.58497634931999 & -0.24082250874502 & 2.10322996469711 \\
\hline $\mathrm{C}$ & -4.13910065004170 & 1.42468839374408 & 0.35680502760371 \\
\hline $\mathrm{H}$ & -3.15811594895927 & 1.88022323156759 & 0.27560195669525 \\
\hline $\mathrm{H}$ & -4.69168250371991 & 1.94001392859481 & 1.14645438397475 \\
\hline $\mathrm{H}$ & -4.67600768743497 & 1.56535776177598 & -0.58546709498910 \\
\hline $\mathrm{C}$ & 0.48248017546425 & 1.18755698353483 & -2.61921781237320 \\
\hline $\mathrm{H}$ & -0.38111064177802 & 1.83715250630721 & -2.48808947892577 \\
\hline $\mathrm{H}$ & 0.77598115802380 & 1.22566079400959 & -3.67408042468238 \\
\hline $\mathrm{C}$ & 1.60669836959366 & 1.68368835453797 & -1.71971973850157 \\
\hline $\mathrm{H}$ & 2.51075461040644 & 1.09371144697277 & -1.90277474671691 \\
\hline $\mathrm{H}$ & 1.84922042557563 & 2.71564698066242 & -1.98776586616063 \\
\hline $\mathrm{N}$ & 1.14561923399675 & 1.58462220664493 & -0.34237894119652 \\
\hline $\mathrm{C}$ & 1.54259412474483 & 2.45835762533586 & 0.55066299208629 \\
\hline $\mathrm{N}$ & 2.76050297007241 & 3.07464086768825 & 0.50272638152456 \\
\hline $\mathrm{C}$ & 2.96294001652578 & 4.45526459700286 & 0.91201608342747 \\
\hline $\mathrm{H}$ & 2.00287838394012 & 4.93863411962913 & 1.06933748613495 \\
\hline $\mathrm{H}$ & 3.49617121851422 & 4.98826045985146 & 0.12096019232042 \\
\hline $\mathrm{H}$ & 3.55286425637859 & 4.52669465722564 & 1.83036095159143 \\
\hline
\end{tabular}




$\begin{array}{llll}\mathrm{C} & 3.94069886433507 & 2.37044061168496 & 0.03298564128352 \\ \mathrm{H} & 3.74914234511739 & 1.30151447007174 & 0.02346600359692 \\ \mathrm{H} & 4.76651916269547 & 2.56551533690646 & 0.72079875576009 \\ \mathrm{H} & 4.24488484090072 & 2.68945834048568 & -0.96818249866900 \\ \mathrm{~N} & 0.71620593003479 & 2.81015717695505 & 1.57257807258090 \\ \mathrm{C} & 1.20115894693782 & 3.03152675512541 & 2.92564884163306 \\ \mathrm{H} & 2.27401707410404 & 2.87204800249328 & 2.96992116667628 \\ \mathrm{H} & 0.72086993051454 & 2.31943778050181 & 3.60120978155630 \\ \mathrm{H} & 0.97482149843295 & 4.04463890651608 & 3.26810643934202 \\ \mathrm{C} & -0.72462220972880 & 2.71263794643258 & 1.43843602942695 \\ \mathrm{H} & -0.98925326240666 & 2.71789616029764 & 0.38523123437725 \\ \mathrm{H} & -1.18384806393755 & 3.57293557013547 & 1.93093648446998 \\ \mathrm{H} & -1.11270452895281 & 1.80018226348780 & 1.89712489165351 \\ \mathrm{C} & 1.01234457399234 & -1.17545414147890 & -2.82366977718518 \\ \mathrm{H} & 2.00673026319394 & -0.73479139250564 & -2.85126342127733 \\ \mathrm{H} & 0.73210287121452 & -1.38627571770003 & -3.86285529131357 \\ \mathrm{C} & 1.06545896742557 & -2.48143432664520 & -2.04725181158702 \\ \mathrm{H} & 1.77928265916526 & -3.16089343539190 & -2.51069754901276 \\ \mathrm{H} & 0.09889907384768 & -2.98497474833579 & -2.03719776942495 \\ \mathrm{~S} & 1.47874509624294 & -2.26166158404241 & -0.28765645133434 \\ \mathrm{C} & 3.19591522859287 & -1.64978919536983 & -0.33866799850712 \\ \mathrm{H} & 3.23722062763839 & -0.78084669560330 & -0.99307969659938 \\ \mathrm{H} & 3.36423408267787 & -1.29812973078602 & 0.67924687881177 \\ \mathrm{C} & 4.21633163111759 & -2.69920425360896 & -0.74628849928143 \\ \mathrm{H} & 4.18803833571247 & -3.55579101968154 & -0.07185249553384 \\ \mathrm{H} & 5.21911838348487 & -2.26730869664655 & -0.71285319395950 \\ \mathrm{H} & 4.04172951755915 & -3.05535811480632 & -1.76260905015685\end{array}$

8.3. Optimized Coordinates of $[2]^{+}$

$\begin{array}{llll}\mathrm{Cu} & -0.02066868611896 & -0.00353738230438 & -0.21719674693897 \\ \mathrm{~N} & -0.13934861456512 & 0.00952207021358 & -2.47941092649463 \\ \mathrm{C} & 0.84379624850358 & 1.02078067960500 & -2.85649290466307 \\ \mathrm{H} & 0.36509195533370 & 1.99763932276271 & -2.79207069235079 \\ \mathrm{H} & 1.18369668795239 & 0.89181293724418 & -3.89481542384174 \\ \mathrm{C} & 2.05956738612561 & 1.01373831939659 & -1.92743035022918 \\ \mathrm{H} & 2.59695443814516 & 0.06984238481167 & -2.06148835052403 \\ \mathrm{H} & 2.74291295386593 & 1.81050392942085 & -2.23827178593246 \\ \mathrm{~N} & 1.64331827680089 & 1.18269423584543 & -0.53987251994483 \\ \mathrm{C} & 2.42778794627239 & 1.77708690647629 & 0.30689744789680 \\ \mathrm{~N} & 3.78169097073479 & 1.95349363157852 & 0.12539998354209 \\ \mathrm{C} & 4.47419944433851 & 3.15589183413655 & 0.55344854118907 \\ \mathrm{H} & 3.75252163166573 & 3.92421913893190 & 0.81679574151232\end{array}$




\begin{tabular}{|c|c|c|c|}
\hline $\mathrm{H}$ & 5.09685009533147 & 3.52512375837867 & -0.26617715946128 \\
\hline 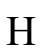 & 5.12462032298800 & 2.97179437563035 & 1.41557852105311 \\
\hline $\mathrm{C}$ & 4.62635838401048 & 0.89276047196243 & -0.39113964796595 \\
\hline $\mathrm{H}$ & 4.06572581502257 & -0.03471320653170 & -0.45687823844832 \\
\hline $\mathrm{H}$ & 5.46576799087430 & 0.73463292074713 & 0.29286312747340 \\
\hline $\mathrm{H}$ & 5.03202128561900 & 1.13064773388654 & -1.37927427945655 \\
\hline $\mathrm{N}$ & 1.91418388984431 & 2.31343521729842 & 1.46644459272180 \\
\hline $\mathrm{C}$ & 2.58028563528556 & 2.08685707864835 & 2.74191764019473 \\
\hline $\mathrm{H}$ & 2.01695241245728 & 1.36131617118952 & 3.33981338276969 \\
\hline $\mathrm{H}$ & 2.65418839187322 & 3.01571457880125 & 3.31163671785674 \\
\hline $\mathrm{H}$ & 3.57885213264877 & 1.69157990950472 & 2.58259972510507 \\
\hline $\mathrm{C}$ & 0.49481909477494 & 2.58072667648965 & 1.56780727314647 \\
\hline $\mathrm{H}$ & 0.11781238838550 & 2.93225184889906 & 0.61180327201955 \\
\hline $\mathrm{H}$ & 0.33495256615331 & 3.34649493653453 & 2.32911350274584 \\
\hline $\mathrm{H}$ & -0.07692907082839 & 1.68648809423511 & 1.84489018119141 \\
\hline $\mathrm{C}$ & -1.52990263961043 & 0.37432220207481 & -2.73331015708846 \\
\hline $\mathrm{H}$ & -2.13334941594037 & -0.53118555784866 & -2.67349651643847 \\
\hline $\mathrm{H}$ & -1.66542006545831 & 0.78994612217420 & -3.74280654391212 \\
\hline $\mathrm{C}$ & -2.05670548989620 & 1.37723027026846 & -1.70488541928769 \\
\hline $\mathrm{H}$ & -1.51265361251283 & 2.31894479189204 & -1.82547742868741 \\
\hline $\mathrm{H}$ & -3.10685416234539 & 1.59001018447914 & -1.92911970977234 \\
\hline $\mathrm{N}$ & -1.89753474071965 & & -0.35242016310504 \\
\hline $\mathrm{C}$ & -2.74070455807846 & 1.18862698680721 & 0.57703647897832 \\
\hline $\mathrm{N}$ & -2.86791912525970 & 0.41150349240354 & 1.70710039374425 \\
\hline $\mathrm{C}$ & -2.91015349102523 & 1.03038346669445 & 3.02544459127472 \\
\hline $\mathrm{H}$ & -1.95395869235690 & 0.88821841099598 & 3.54228536159037 \\
\hline $\mathrm{H}$ & -3.69847915376866 & 0.58568311660603 & 3.63663110074922 \\
\hline $\mathrm{H}$ & -3.09182496015981 & 2.09698596429732 & 2.93760245003152 \\
\hline $\mathrm{C}$ & -2.37767738000175 & -0.95102774824422 & 1.69965948498916 \\
\hline $\mathrm{H}$ & -2.56497220022312 & -1.40457494686310 & 0.73086380101650 \\
\hline $\mathrm{H}$ & -2.89815256952679 & -1.51504301632108 & 2.47572594416580 \\
\hline $\mathrm{H}$ & -1.29865145748380 & -1.00497935241439 & 1.89062766736292 \\
\hline $\mathrm{N}$ & -3.57634563698908 & 2.28199252464515 & 0.51526642002142 \\
\hline $\mathrm{C}$ & -4.92781562583146 & 2.26314545546429 & 1.04640338994966 \\
\hline $\mathrm{H}$ & -5.22069390214681 & 1.24254072790409 & 1.27732268204653 \\
\hline $\mathrm{H}$ & -5.61556044031062 & 2.66444455066177 & 0.29680746918644 \\
\hline $\mathrm{H}$ & -5.02333236038136 & 2.87157018899781 & 1.95235388079064 \\
\hline $\mathrm{C}$ & -3.11073977894397 & 3.56893978184952 & 0.03211333557552 \\
\hline $\mathrm{H}$ & -2.03334450727627 & 3.55223126695613 & -0.10079395669616 \\
\hline $\mathrm{H}$ & -3.34947972822986 & 4.33823993652272 & 0.77253685827414 \\
\hline $\mathrm{H}$ & -3.57864092351292 & 3.85065759961126 & -0.91608056980558 \\
\hline $\mathrm{C}$ & 0.21579594670859 & -1.36060425577116 & -2.83593891753334 \\
\hline $\mathrm{H}$ & 1.30294802260998 & -1.43324965962385 & -2.85533333301816 \\
\hline $\mathrm{H}$ & -0.14716198364959 & -1.62932243255309 & -3.83918813810115 \\
\hline $\mathrm{C}$ & -0.31866016440920 & -2.37346922659614 & -1.82161726215918 \\
\hline $\mathrm{H}$ & -1.41221273644063 & -2.36684077483611 & -1.86297207613981 \\
\hline
\end{tabular}




$\begin{array}{llll}\mathrm{H} & 0.00165301122458 & -3.37500030540004 & -2.12584695332909 \\ \mathrm{~N} & 0.15221801657977 & -2.04870906755242 & -0.48000547220817 \\ \mathrm{C} & 0.35391320807660 & -2.99597928346036 & 0.38527190646110 \\ \mathrm{~N} & 1.17127310774183 & -2.77654158850885 & 1.47099706499243 \\ \mathrm{C} & 0.76733811157042 & -3.20997147250957 & 2.80165240210526 \\ \mathrm{H} & 0.45900639969325 & -2.34773645266213 & 3.40376103605743 \\ \mathrm{H} & 1.59378659921316 & -3.70719246314668 & 3.31425596187716 \\ \mathrm{H} & -0.07139749358356 & -3.89633069737145 & 2.73849716104013 \\ \mathrm{C} & 2.10264806349867 & -1.66847813565355 & 1.45558930974954 \\ \mathrm{H} & 2.51333788034841 & -1.54561167524769 & 0.45793964502804 \\ \mathrm{H} & 2.90868896140009 & -1.87798150436208 & 2.16112997610051 \\ \mathrm{H} & 1.62551648999683 & -0.72198000614111 & 1.73800535357123 \\ \mathrm{~N} & -0.17875679502565 & -4.26290833119582 & 0.29282574987475 \\ \mathrm{C} & -1.55760976581968 & -4.47953423891133 & -0.10393237274058 \\ \mathrm{H} & -2.08881999610624 & -3.53347744524514 & -0.14316946427505 \\ \mathrm{H} & -2.04981267935930 & -5.11462188517722 & 0.63866400918140 \\ \mathrm{H} & -1.63769219707114 & -4.96970218688687 & -1.07901063476870 \\ \mathrm{C} & 0.55269280694027 & -5.45015785706267 & 0.69828063979571 \\ \mathrm{H} & 1.59404366909819 & -5.19913843777003 & 0.88048375216408 \\ \mathrm{H} & 0.50684721978458 & -6.19472051250851 & -0.10164065431725 \\ \mathrm{H} & 0.13385094147460 & -5.90099421784626 & 1.60455984147149\end{array}$

\subsection{Optimized Coordinates of $\left[2 \cdot \mathrm{O}_{2}\right]^{+}$}

$\begin{array}{llll}\mathrm{Cu} & 0.01067962901335 & -0.00852962323727 & -0.04193123449543 \\ \mathrm{O} & -0.09835855050202 & -0.02871037427114 & 1.94286615744684 \\ \mathrm{O} & 0.97767745561778 & 0.06245096679442 & 2.65198179260781 \\ \mathrm{~N} & -0.10367247749261 & 0.01582010038053 & -2.23324465368407 \\ \mathrm{C} & 0.97202496107886 & 0.91590041338599 & -2.68796875054211 \\ \mathrm{H} & 0.59191794715767 & 1.93642532076130 & -2.66850562996035 \\ \mathrm{H} & 1.25637280083811 & 0.68310306478819 & -3.72073391554423 \\ \mathrm{C} & 2.17414612277534 & 0.82948524813185 & -1.75789582386131 \\ \mathrm{H} & 2.60788203144762 & -0.17481028898564 & -1.81444775090348 \\ \mathrm{H} & 2.94052943351158 & 1.52789948933894 & -2.10354036654364 \\ \mathrm{~N} & 1.72056214444567 & 1.14464515583367 & -0.41038287713791 \\ \mathrm{C} & 2.46761664687562 & 1.88048822069543 & 0.37057026776295 \\ \mathrm{~N} & 3.83295903494259 & 1.95391640987427 & 0.26354063440314 \\ \mathrm{C} & 4.57145815073077 & 3.18783153884494 & 0.47225244139189 \\ \mathrm{H} & 3.88282376855185 & 4.02455562923785 & 0.54985867928040 \\ \mathrm{H} & 5.23300253756995 & 3.35830103158407 & -0.38142617502287 \\ \mathrm{H} & 5.18587443308683 & 3.14907829826823 & 1.37708628313019 \\ \mathrm{C} & 4.63730121814981 & 0.79429285628031 & -0.07339242904558 \\ \mathrm{H} & 4.03171061390512 & -0.10357795814947 & -0.02238948243005\end{array}$




\begin{tabular}{|c|c|c|c|}
\hline $\mathrm{H}$ & 5.45271608427972 & 0.70115001622216 & 0.64908100393837 \\
\hline $\mathrm{H}$ & 5.07405411534715 & 0.87196843907248 & -1.07356381813712 \\
\hline & 1.89874132060994 & 2.66111510114593 & 1.33071560118052 \\
\hline $\mathrm{C}$ & 2.49873879192613 & 2.84329237473132 & 2.64236606158315 \\
\hline $\mathrm{H}$ & 1.81179817738912 & 2.47447568585913 & 3.40738321975344 \\
\hline $\mathrm{H}$ & 2.70913321347286 & 3.89813391589183 & 2.83882608135965 \\
\hline $\mathrm{H}$ & 3.42207135805837 & 2.27682442816692 & 2.71409095048457 \\
\hline $\mathrm{C}$ & 0.52784629509259 & 3.11128570780888 & 1.20808845902986 \\
\hline $\mathrm{H}$ & 0.24399519854781 & 3.10848845505129 & 0.16015708145489 \\
\hline $\mathrm{H}$ & 0.45469370321609 & 4.12822037829255 & 1.60102069200445 \\
\hline $\mathrm{H}$ & -0.16522425753510 & 2.47154534849978 & 1.75946366971738 \\
\hline $\mathrm{C}$ & -1.44446243122649 & 0.52023046344613 & -2.58642473343241 \\
\hline $\mathrm{H}$ & -2.13498362650958 & -0.32191323999229 & -2.58256090856165 \\
\hline $\mathrm{H}$ & -1.44038751064045 & 0.94650008351606 & -3.59626721154917 \\
\hline $\mathrm{C}$ & -1.92461879919268 & 1.54227754728088 & -1.56656913342880 \\
\hline $\mathrm{H}$ & -1.28084903592489 & 2.42744807425360 & -1.61149030162217 \\
\hline $\mathrm{H}$ & -2.93322619596346 & 1.86837010260872 & -1.83544782042840 \\
\hline $\mathrm{N}$ & -1.88591031237088 & 0.91486930404204 & -0.25502621178175 \\
\hline $\mathrm{C}$ & -2.81956299293446 & 1.15982446600477 & 0.62382619909998 \\
\hline $\mathrm{N}$ & -3.12004626793887 & 0.23164526483672 & 1.57936192586419 \\
\hline $\mathrm{C}$ & -3.35803773204341 & 0.60238523737361 & 2.96430687080709 \\
\hline $\mathrm{H}$ & -2.57291669690372 & 0.17491824875759 & 3.59454102756161 \\
\hline $\mathrm{H}$ & -4.32556113922789 & 0.23096096609741 & 3.31281778575113 \\
\hline $\mathrm{H}$ & -3.33442870434847 & 1.68260932419495 & 3.07434042592293 \\
\hline $\mathrm{C}$ & -2.88796057864095 & -1.17936505236747 & 1.34777783726780 \\
\hline $\mathrm{H}$ & -2.87293418420817 & -1.36691297658539 & 0.27822327564864 \\
\hline $\mathrm{H}$ & -3.70194300821099 & -1.74928116072535 & 1.80320706297986 \\
\hline $\mathrm{H}$ & -1.94136022807059 & -1.51326467795498 & 1.77763909689014 \\
\hline $\mathrm{N}$ & -3.57685163980970 & 2.30332931552859 & 0.64220213676318 \\
\hline $\mathrm{C}$ & -4.97536945584352 & 2.30770902165676 & 1.03817231478179 \\
\hline $\mathrm{H}$ & -5.34365234754087 & 1.28848454795803 & 1.11199682510914 \\
\hline $\mathrm{H}$ & -5.55962312100936 & 2.83828254762794 & 0.28168346519350 \\
\hline $\mathrm{H}$ & -5.12654149028965 & 2.81038868222150 & 1.99861715682531 \\
\hline $\mathrm{C}$ & -3.02301709606427 & 3.60220166044788 & 0.30659743144992 \\
\hline $\mathrm{H}$ & -1.94605844014877 & 3.53473588677769 & 0.20719888823137 \\
\hline $\mathrm{H}$ & -3.24972577560008 & 4.30871931622268 & 1.10991894654752 \\
\hline $\mathrm{H}$ & -3.44105791766214 & 3.99715178644437 & -0.62391252083194 \\
\hline $\mathrm{C}$ & 0.10239320514359 & -1.37290761537383 & -2.68530026913023 \\
\hline $\mathrm{H}$ & 1.17443654494180 & -1.55730916005631 & -2.73868257832660 \\
\hline $\mathrm{H}$ & -0.31365076674015 & -1.51498263423279 & -3.68947017804808 \\
\hline $\mathrm{C}$ & -0.50757362673388 & -2.35426263568339 & -1.69476584350445 \\
\hline $\mathrm{H}$ & -1.59464706694761 & -2.22129388658912 & -1.67420637915361 \\
\hline $\mathrm{H}$ & -0.31747563113720 & -3.37461035361740 & -2.03884419695101 \\
\hline $\mathrm{N}$ & 0.09234974044536 & -2.09702648038167 & -0.39529089662396 \\
\hline $\mathrm{C}$ & 0.38234969032960 & -3.08453221548448 & 0.40859954195558 \\
\hline $\mathrm{N}$ & 1.40991052353398 & -2.95497475072868 & 1.29672739607251 \\
\hline
\end{tabular}




$\begin{array}{llll}\mathrm{C} & 1.29954565587591 & -3.40319753266938 & 2.67447273269753 \\ \mathrm{H} & 1.37984702249041 & -2.54305962897777 & 3.34481119151357 \\ \mathrm{H} & 2.08845666871749 & -4.11745680969291 & 2.92635196235614 \\ \mathrm{H} & 0.33368409335966 & -3.87344377159416 & 2.83751135064664 \\ \mathrm{C} & 2.54696106961758 & -2.10519928166507 & 0.99338772755147 \\ \mathrm{H} & 2.64001983648776 & -2.00667026755084 & -0.08498911999327 \\ \mathrm{H} & 3.45011629362384 & -2.57786505623754 & 1.38844079323513 \\ \mathrm{H} & 2.44407319427446 & -1.11341432768634 & 1.43567128551059 \\ \mathrm{~N} & -0.25965270044761 & -4.29524225893515 & 0.40614532480163 \\ \mathrm{C} & -1.67891862663043 & -4.41996894648952 & 0.13072479706440 \\ \mathrm{H} & -2.14069891388723 & -3.44051679480991 & 0.09942511235418 \\ \mathrm{H} & -2.15127609953546 & -4.99782795752670 & 0.92988725538816 \\ \mathrm{H} & -1.86692608619673 & -4.93128445266008 & -0.81780695634269 \\ \mathrm{C} & 0.42698064045089 & -5.54311665517901 & 0.69754873973085 \\ \mathrm{H} & 1.50023430082717 & -5.37901053449124 & 0.72538279586245 \\ \mathrm{H} & 0.20279457929784 & -6.26744669094578 & -0.08994624989664 \\ \mathrm{H} & 0.10734128505468 & -5.96984939071212 & 1.65324866094951\end{array}$

\section{Reactivity and Kinetic Studies}

The substrates TEMPO-H (10, 20, 30, 80 mmols) and TEMPO-D (40, 60, 120, 300 mmols) were added to $0.4 \mathrm{mM}$ solutions of $\left[1 \cdot \mathrm{O}_{2}\right] \mathrm{B}\left(\mathrm{C}_{6} \mathrm{~F}_{5}\right)_{4}$ via syringe and allowed to react at $-135^{\circ} \mathrm{C}$. Decay of the superoxide band at $742 \mathrm{~nm}$ was monitored over time and the pseudo-first-order rate constants were calculated from the fitted exponential slopes. A plot of $k_{\text {obs }}$ vs substrate concentration was used to determine the second order rate constant. See Figures S6-S9 and Table S4 for details.

The same procedure mentioned above was followed for the reactions of TEMPO-H $(20,30,40$, 50 mmols) with $0.4 \mathrm{mM}$ solutions of $\left[2 \cdot \mathrm{O}_{2}\right] \mathrm{B}\left(\mathrm{C}_{6} \mathrm{~F}_{5}\right)_{4}$. See figures $\mathbf{S 1 0 - S 1 1}$ and Table S5 for details. 


\section{$\left[1 \cdot \mathrm{O}_{2}\right] \mathrm{B}\left(\mathrm{C}_{6} \mathrm{~F}_{5}\right)_{4}$ with TEMPO-H:}

a)

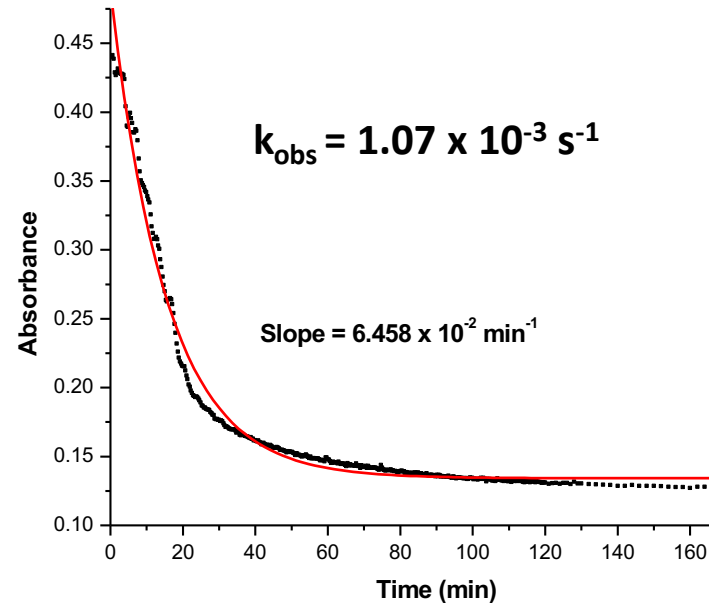

c)

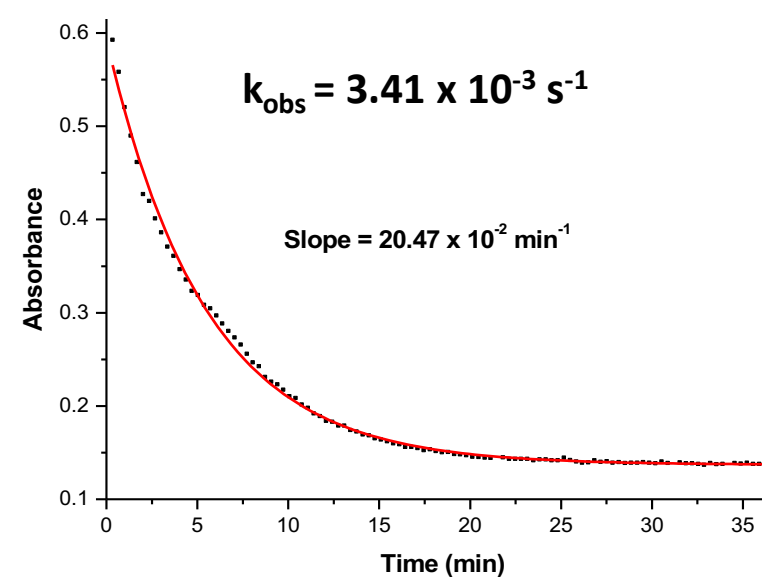

b)

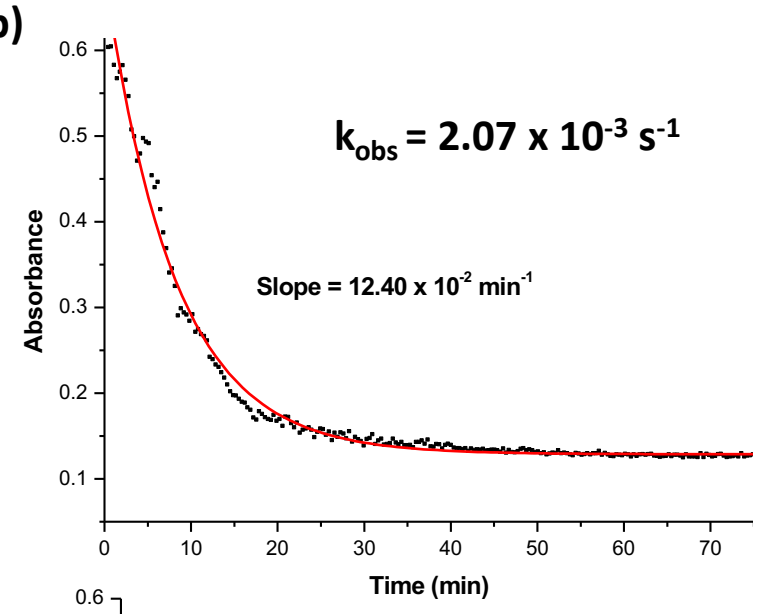

d)

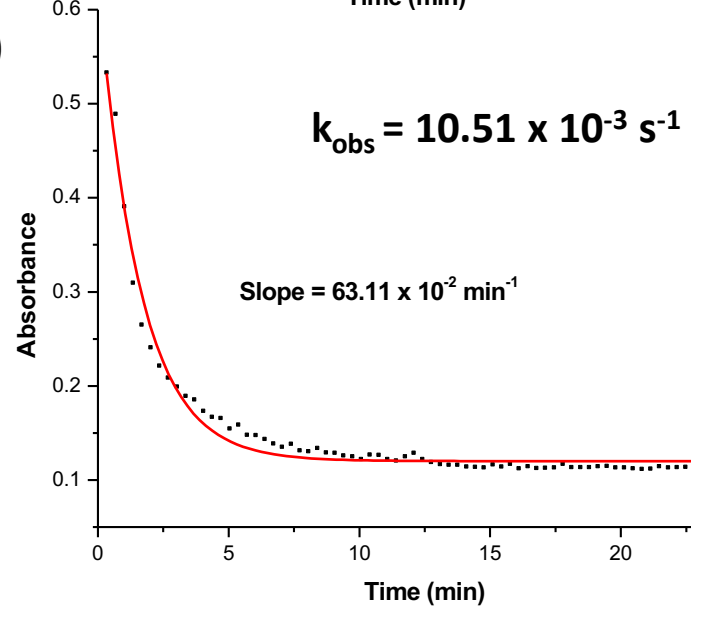

Figure S6. Representative exponential plots of absorbance at $742 \mathrm{~nm}$ versus time for the reaction of $\sim 0.4 \mathrm{mM}$ $\left[\mathbf{1} \cdot \mathrm{O}_{2}\right] \mathrm{B}\left(\mathrm{C}_{6} \mathrm{~F}_{5}\right)_{4}$ at $-135^{\circ} \mathrm{C}$ in 2-MeTHF with (a) 10 , (b) 20, (c) 30 and (d) 80 mmols of TEMPO-H to determine $k_{\text {obs }}$ at each concentration. Black dots represent the actual absorbances recorded and the red line is the fitted trace. 
Table S4. Table containing the $k_{\mathrm{obs}}\left(\mathrm{s}^{-1}\right)$ values for the reaction of $0.4 \mathrm{mM}\left[\mathbf{1} \cdot \mathrm{O}_{2}\right] \mathrm{B}\left(\mathrm{C}_{6} \mathrm{~F}_{5}\right)_{4}$ at $-135{ }^{\circ} \mathrm{C}$ in $2-\mathrm{MeTHF}$ with 10, 20, 30 and 80 mmols of TEMPO-H.

\begin{tabular}{lllll}
\hline \multirow{2}{*}{ mol } & \multicolumn{3}{c}{$\boldsymbol{k}_{\text {obs s }}{ }^{-1}$} & Average \\
\cline { 2 - 4 } & Trial 1 & Trial 2 & Trial 3 & \\
\hline 1.00E-02 & $1.06 \mathrm{E}-03$ & $1.07 \mathrm{E}-03$ & $0.97 \mathrm{E}-03$ & $1.03 \mathrm{E}-03$ \\
$2.00 \mathrm{E}-02$ & $2.07 \mathrm{E}-03$ & $2.30 \mathrm{E}-03$ & $2.09 \mathrm{E}-03$ & $2.15 \mathrm{E}-03$ \\
$3.00 \mathrm{E}-02$ & $3.10 \mathrm{E}-03$ & $3.41 \mathrm{E}-03$ & $4.25 \mathrm{E}-03$ & $3.57 \mathrm{E}-03$ \\
$8.00 \mathrm{E}-02$ & $0.75 \mathrm{E}-02$ & $1.05 \mathrm{E}-02$ & $1.36 \mathrm{E}-02$ & $1.05 \mathrm{E}-02$ \\
\hline
\end{tabular}

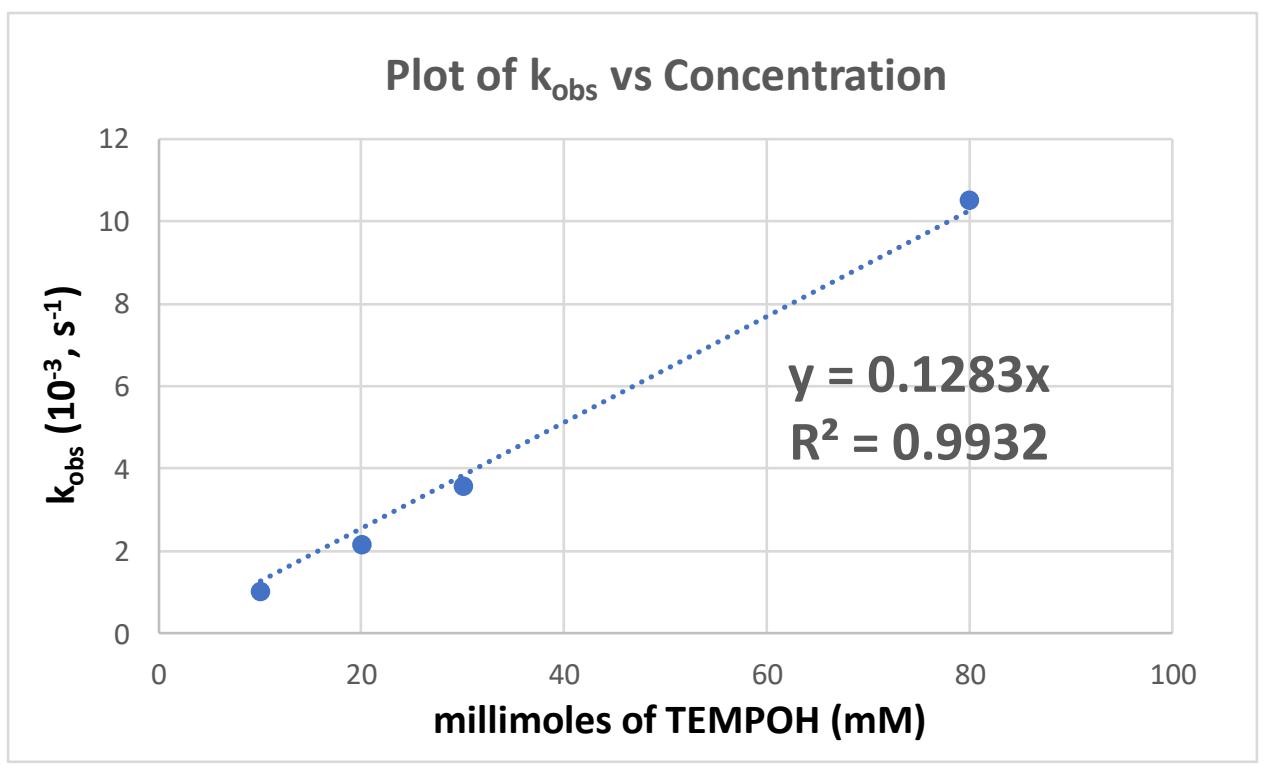

Figure S7. Plot for $\left[\mathbf{1}^{\cdot} \mathrm{O}_{2}\right] \mathrm{B}\left(\mathrm{C}_{6} \mathrm{~F}_{5}\right)_{4}$ of pseudo-first order rate constants $\left(k_{\mathrm{obs}}\right)$ against the TEMPO-H concentrations; from the slope of the linear fit, the second-order rate constant is calculated to be $1.28 \times 10^{-1} \mathrm{M}^{-1} \mathrm{~s}^{-1}$. 


\section{$\left[1 \cdot \mathrm{O}_{2}\right] \mathrm{B}\left(\mathrm{C}_{6} \mathrm{~F}_{5}\right)_{4}$ with TEMPO-D:}

a)

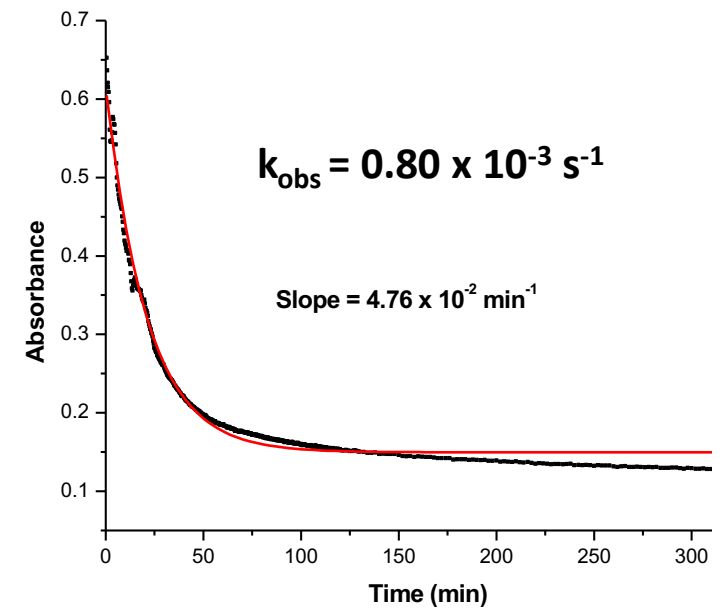

c)

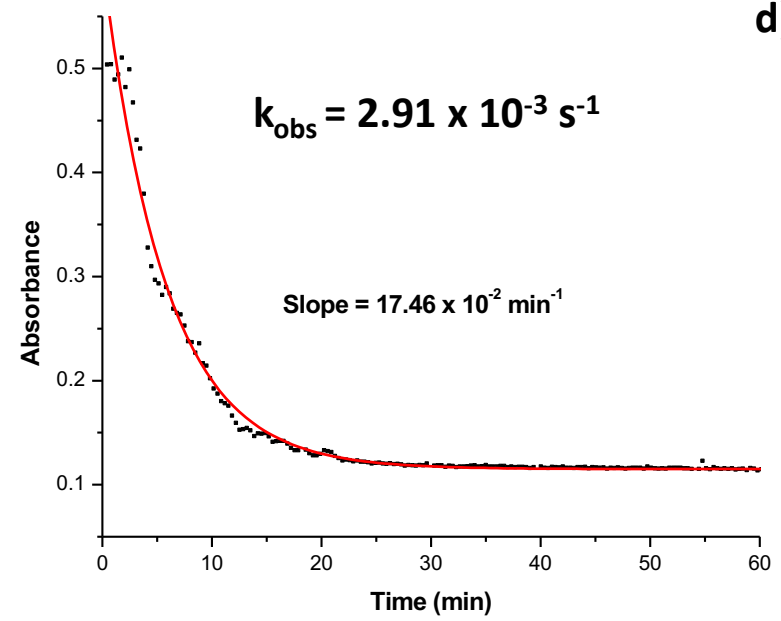

b)

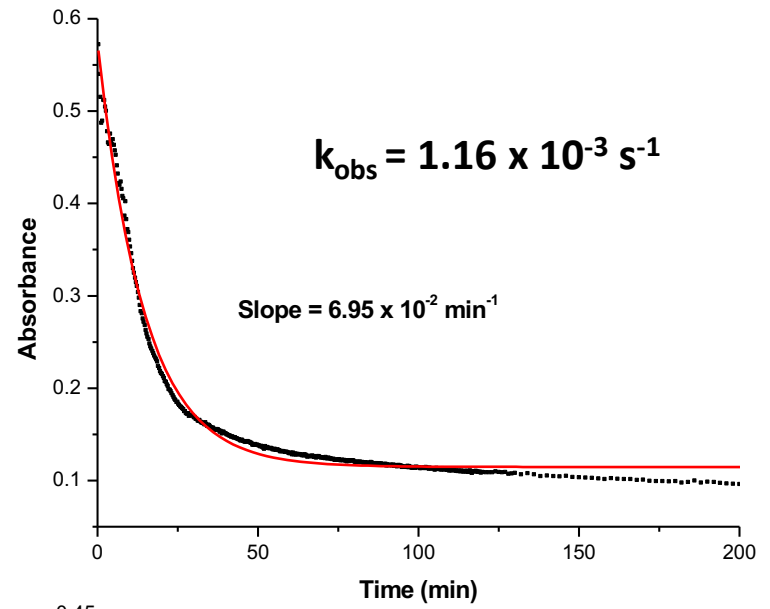

d)

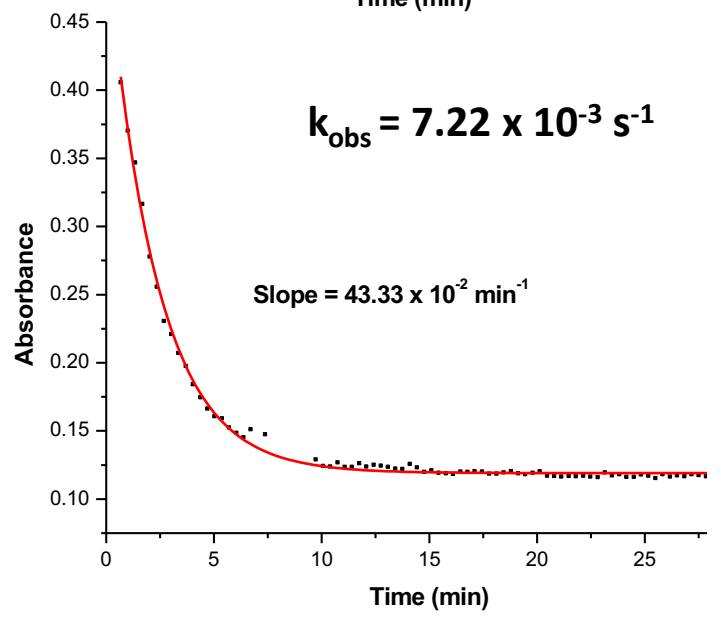

Figure S8. Representative exponential plots of absorbance at $742 \mathrm{~nm}$ versus time for the reaction of $\sim 0.4 \mathrm{mM}$ $\left[\mathbf{1} \cdot \mathrm{O}_{2}\right] \mathrm{B}\left(\mathrm{C}_{6} \mathrm{~F}_{5}\right)_{4}$ at $-135^{\circ} \mathrm{C}$ in 2-MeTHF with (a) 40, (b) 60, (c) 120 and (d) 300 mmols of TEMPO-D to determine $k_{\text {obs }}$ at each concentration. Black dots represent the actual absorbances recorded and the red line is the fitted trace. 


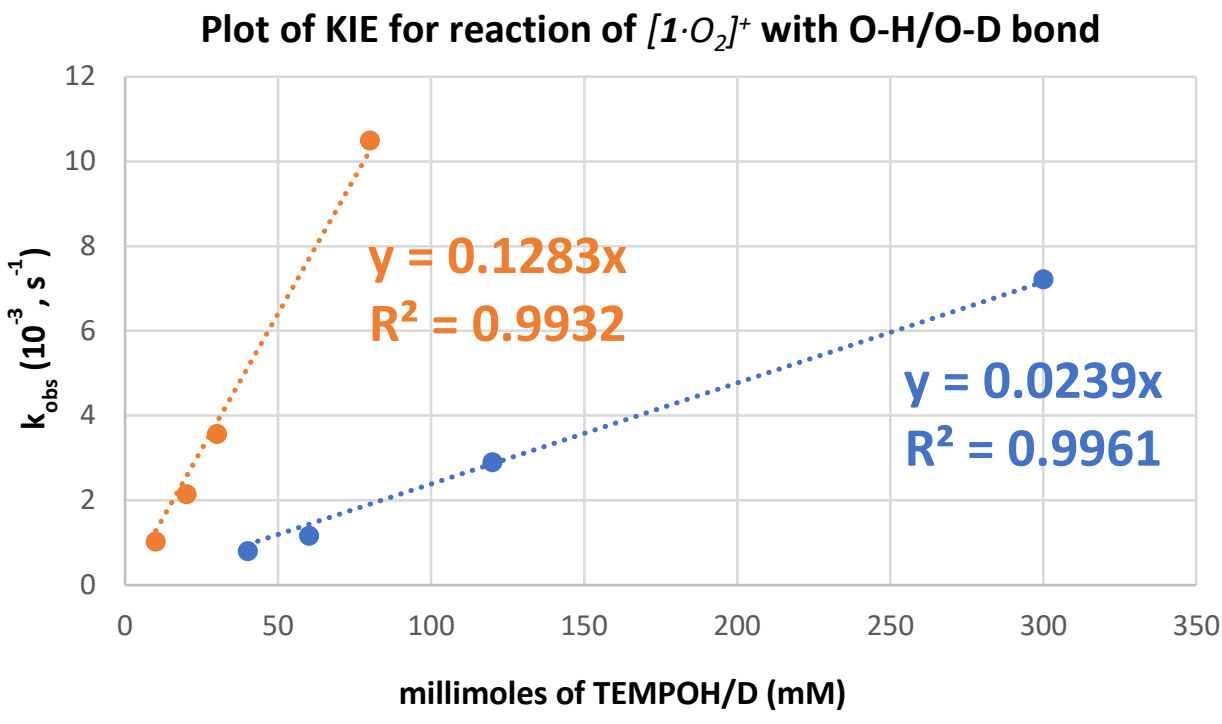

Figure S9. Plots of pseudo first order rate constants ( $\left.k_{\mathrm{obs}}\right)$ plotted against various concentrations of TEMPO-H(D) to obtain the second order rate constant, $k_{\mathrm{H}}=1.28 \times 10^{-1} \mathrm{M}^{-1} \mathrm{sec}^{-1}$ (orange circle) and $k_{\mathrm{D}}=0.24 \times 10^{-1} \mathrm{M}^{-1} \mathrm{sec}^{-1}$ (blue circle) thus yielding a primary $\mathrm{KIE}$ of 5.4 in $2-\mathrm{MeTHF}$ at $-135^{\circ} \mathrm{C}$, for $\mathrm{H}$-atom abstraction by $\left[\mathbf{1}^{\cdot} \mathrm{O}_{2}\right] \mathrm{B}\left(\mathrm{C}_{6} \mathrm{~F}_{5}\right) 4$. 
a)

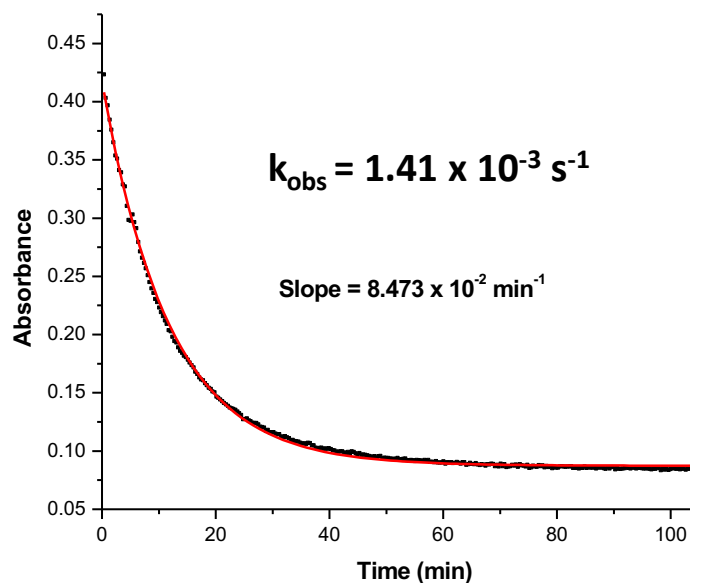

c)

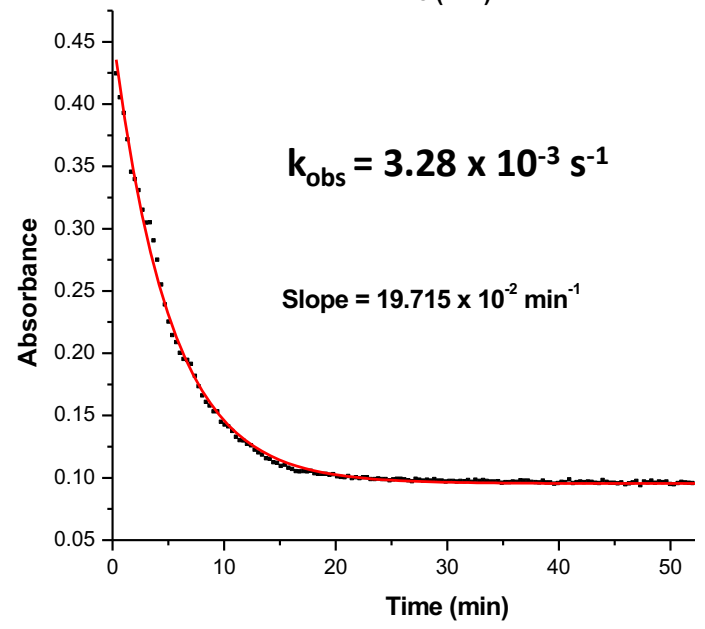

b)

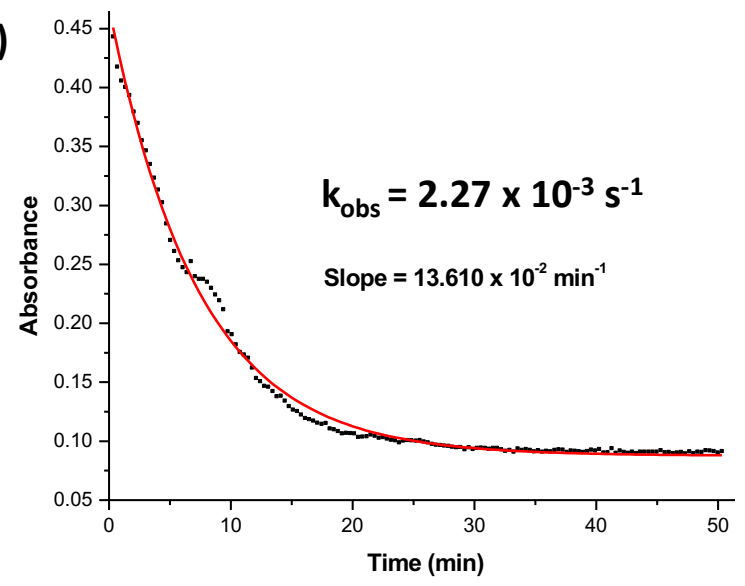

d)

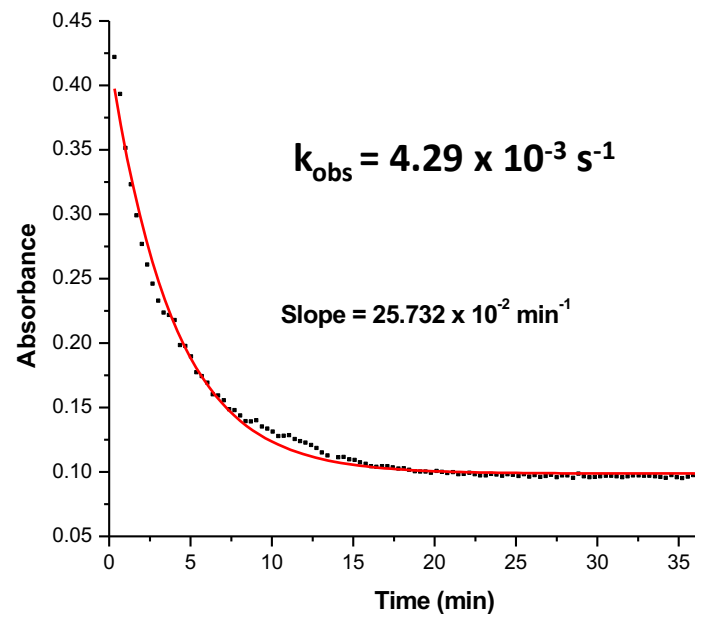

Figure S10. Representative exponential plots of absorbance at $780 \mathrm{~nm}$ versus time for the reaction of $\sim 0.4 \mathrm{mM}$ [2. $\left.\mathrm{O}_{2}\right] \mathrm{B}\left(\mathrm{C}_{6} \mathrm{~F}_{5}\right)_{4}$ at $-135^{\circ} \mathrm{C}$ in MeTHF with (a) 20, (b) 30, (c) 40 and (d) 50 mmols of TEMPO-H to determine $k_{\text {obs }}$ at each concentration. Black dots represent the actual absorbances recorded and the red line is the fitted trace. 
Table S5. Table containing the $k_{\mathrm{obs}}\left(\mathrm{s}^{-1}\right)$ values for the reaction of $\sim 0.4 \mathrm{mM}\left[2 \cdot \mathrm{O}_{2}\right] \mathrm{B}\left(\mathrm{C}_{6} \mathrm{~F}_{5}\right)_{4}$ at $135^{\circ} \mathrm{C}$ in 2-MeTHF with $20,30,40$ and 50 mmols of TEMPO-H.

\begin{tabular}{lcccc}
\hline \multirow{2}{*}{ mol } & \multicolumn{3}{c}{$\boldsymbol{k}_{\text {obs s }}{ }^{-1}$} & Average \\
\cline { 2 - 4 } & Trial 1 & Trial 2 & Trial 3 & \\
\hline 2.00E-02 & $1.26 \mathrm{E}-03$ & $1.41 \mathrm{E}-03$ & $1.55 \mathrm{E}-03$ & $1.41 \mathrm{E}-03$ \\
$3.00 \mathrm{E}-02$ & $2.27 \mathrm{E}-03$ & $2.61 \mathrm{E}-03$ & $2.24 \mathrm{E}-03$ & $2.37 \mathrm{E}-03$ \\
$4.00 \mathrm{E}-02$ & $3.01 \mathrm{E}-03$ & $3.49 \mathrm{E}-03$ & $3.28 \mathrm{E}-03$ & $3.26 \mathrm{E}-03$ \\
$5.00 \mathrm{E}-02$ & $4.01 \mathrm{E}-03$ & $4.45 \mathrm{E}-03$ & $4.29 \mathrm{E}-03$ & $4.25 \mathrm{E}-03$ \\
\hline
\end{tabular}

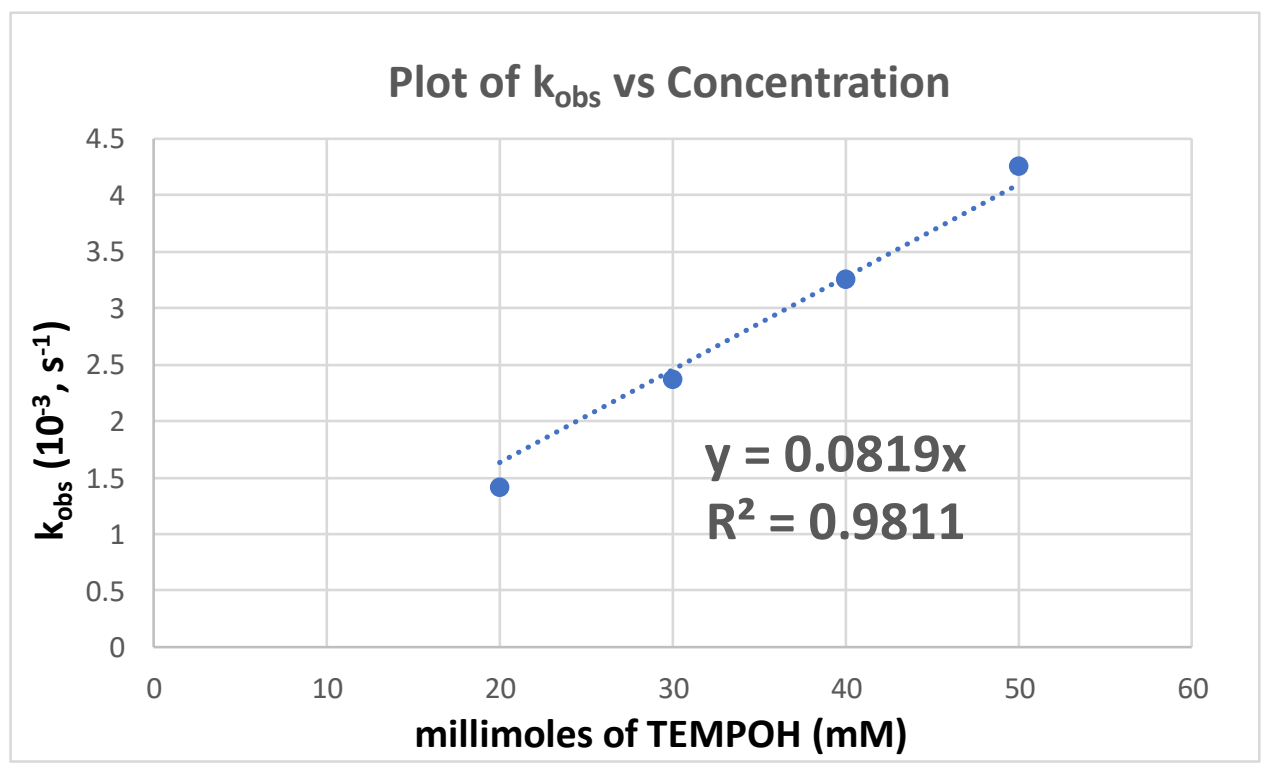

Figure S11. Plot $\left\{\right.$ for $\left.\left[2 \cdot \mathrm{O}_{2}\right] \mathrm{B}\left(\mathrm{C}_{6} \mathrm{~F}_{5}\right)_{4}\right\}$ of pseudo first order rate constants $\left(k_{\mathrm{obs}}\right)$ against the TEMPO-H concentrations; from the slope of the linear fit, the second-order rate constant is calculated to be $0.82 \times 10^{-1} \mathrm{M}^{-1} \mathrm{~s}^{-1}$. 


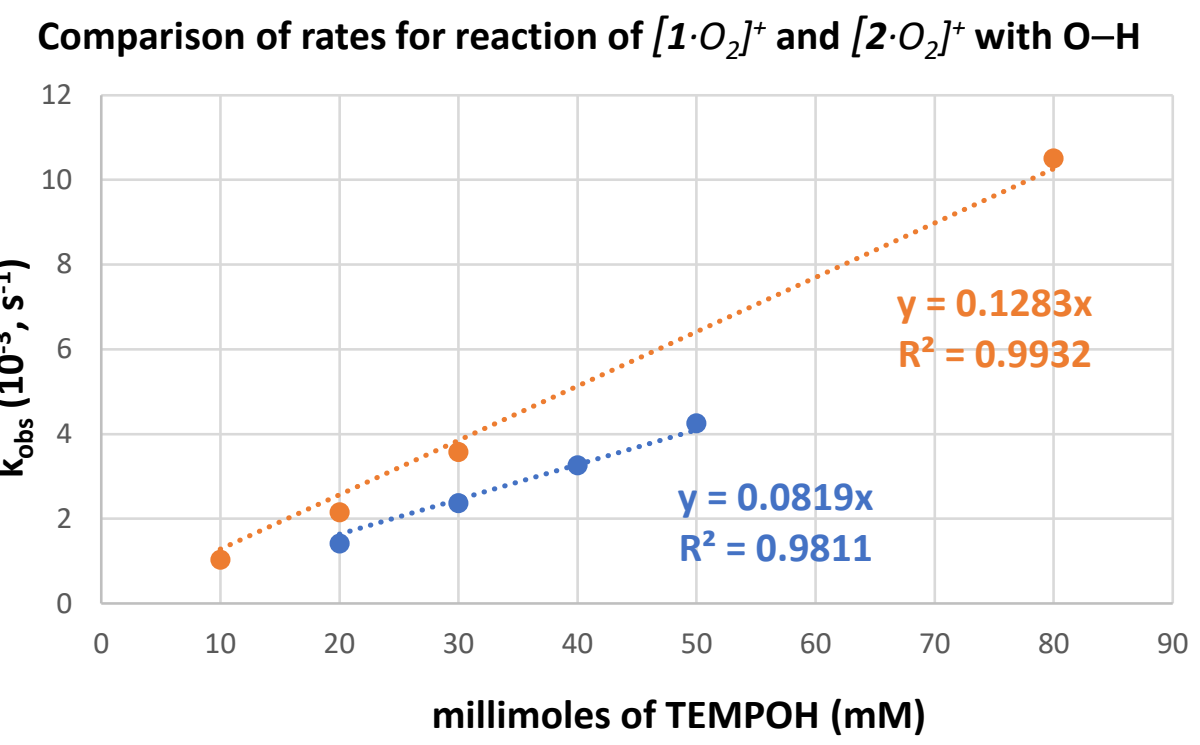

Figure S12. Comparative analysis of rates of hydrogen atom abstraction reactivity with TEMPO-H (2-MeTHF at $\left.135^{\circ} \mathrm{C}\right): k_{2}=1.28 \times 10^{-1} \mathrm{M}^{-1} \mathrm{~s}^{-1}$, in orange, $\left[\mathbf{1} \cdot \mathrm{O}_{2}\right] \mathrm{B}\left(\mathrm{C}_{6} \mathrm{~F}_{5}\right)_{4} ; 0.82 \times 10^{-1} \mathrm{M}^{-1} \mathrm{~s}^{-1}$, in blue, $\left[2 \cdot \mathrm{O}_{2}\right] \mathrm{B}\left(\mathrm{C}_{6} \mathrm{~F}_{5}\right)_{4}$.

\section{Product Analyses}

\section{A) Quantification of Hydroperoxo Product}

The formation (and the yield) of the final cupric hydroperoxo product was further verified by acidification (i.e. liberation of $\mathrm{H}_{2} \mathrm{O}_{2}$ ) and subsequent quantification of triodide $\left(\mathrm{I}_{3}{ }^{-}\right.$) formed when treated with a MeCN-saturated solution of $\mathrm{I}^{-}$. In a typical experiment, $2.5 \mathrm{ml}$ of a $0.80 \mathrm{mM}$ solution of $[1] \mathrm{B}\left(\mathrm{C}_{6} \mathrm{~F}_{5}\right)_{4}$ in 2-MeTHF was taken in a cuvette in the glovebox, and oxygenated at $-135^{\circ} \mathrm{C}$. The superoxide generated was mixed with TEMPO-H and at the end of the HAA reaction, 1 equivalent of $\mathrm{HCl} \cdot \mathrm{Et}_{2} \mathrm{O}\left(2.0 \mathrm{M}\right.$, Sigma Aldrich) was added via a syringe (to release $\mathrm{H}_{2} \mathrm{O}_{2}$ ) until complete disappearance of the hydroperoxide features is noted via UV-Vis spectroscopy. Then, $150 \mu \mathrm{L}$ of solution in the cuvette was transferred into a cuvette with saturated NaI solution in $\mathrm{MeCN}(2.0 \mathrm{~mL})$ at RT. This mixture was allowed to incubate for 6 mins. The UV-Vis spectrum of this solution was then taken showing the formation of triodide $\left(\mathrm{I}_{3}{ }^{-}\right)$at $362 \mathrm{~nm}$ (Figure S13). The 
yield of $\mathrm{H}_{2} \mathrm{O}_{2}$ was found to be $94 \%$ when compared with standard series of solutions (see Figure S14). ${ }^{22}$ Note: (1)Without acidification, $\left[1 \cdot \mathrm{O}_{2}\right] \mathrm{B}\left(\mathrm{C}_{6} \mathrm{~F}_{5}\right)_{4}$ by itself did not show any presence of peroxide formation upon warming and (2) the protonation of the cupric superoxide complex $\left[1 \cdot \mathrm{O}_{2}\right] \mathrm{B}\left(\mathrm{C}_{6} \mathrm{~F}_{5}\right)_{4}$ resulted in $43 \%$ yield of $\mathrm{H}_{2} \mathrm{O}_{2}$, consistent with previous observation of disproportionation of protonated superoxo radical: $2 \mathrm{H}^{+}+2 \mathrm{O}_{2}{ }^{--} \rightarrow \mathrm{H}_{2} \mathrm{O}_{2}+\mathrm{O}_{2} .^{22}$

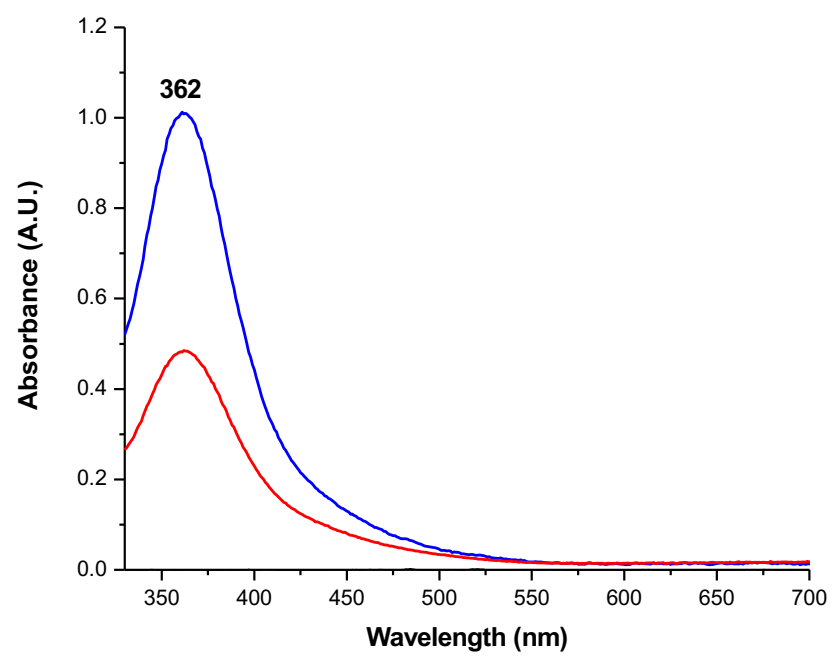

Figure S13. UV-Vis spectra of $\mathrm{I}_{3}{ }^{-}$generated $\left(\lambda_{\max }=362 \mathrm{~nm}\right)$ from the released $\mathrm{H}_{2} \mathrm{O}_{2}$ upon addition of 1 equiv of $\mathrm{H}^{+}$ to the reaction mixture containing $[\mathbf{1} \cdot \mathrm{OOH}] \mathrm{B}\left(\mathrm{C}_{6} \mathrm{~F}_{5}\right)_{4}$ species, in blue and $\left[\mathbf{1} \cdot \mathrm{O}_{2}\right] \mathrm{B}\left(\mathrm{C}_{6} \mathrm{~F}_{5}\right)_{4}$ complex (control), in red. 

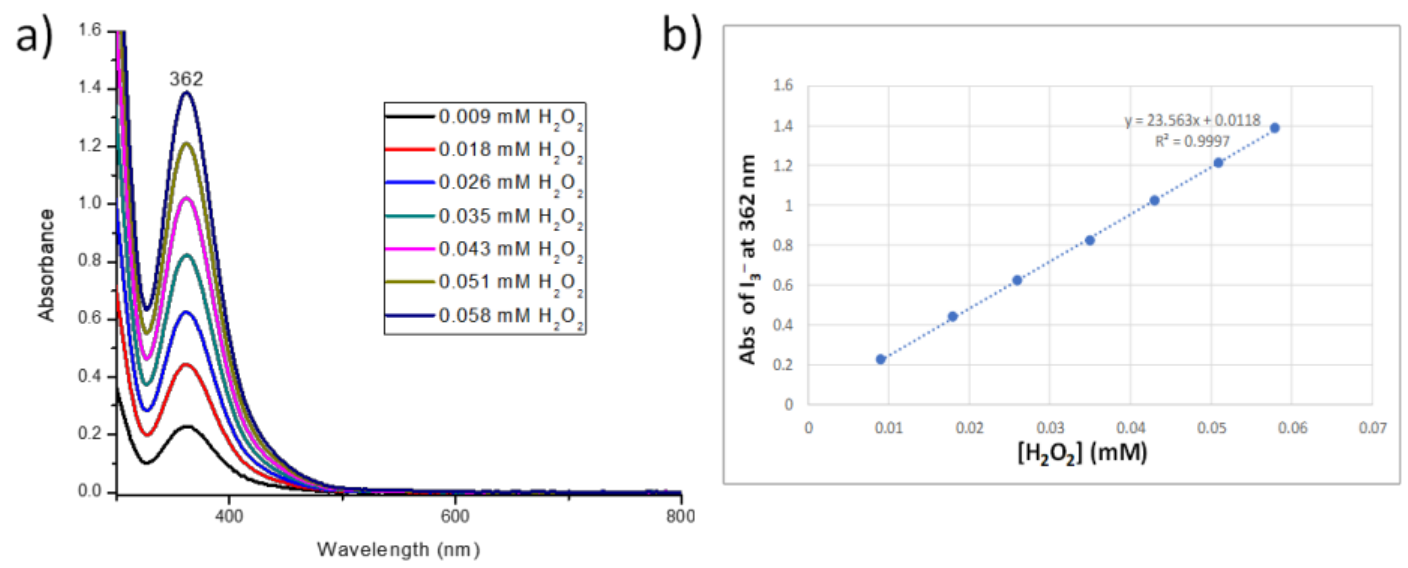

Figure S14. UV-Visible spectrum of triodide $\left(\mathrm{I}_{3}{ }^{-}\right)$at $362 \mathrm{~nm}$ obtained on spectrophotometric titration of $\mathrm{I}_{3}{ }^{-}$with $\mathrm{H}_{2} \mathrm{O}_{2}$ solutions to generate b) the standard linear curve. See J. Am. Chem. Soc. 2018, 140, 9042-9045 for details. ${ }^{22}$

\section{B) EPR Data}

In the glovebox, a $500 \mathrm{ml}$ of $0.8 \mathrm{mM}$ solution of $[1] \mathrm{B}\left(\mathrm{C}_{6} \mathrm{~F}_{5}\right)_{4}$ in 4:1 (v/v) 2-MeTHF:THF was prepared, transferred to an EPR tube and capped with tightfitting septa. The sample tube was placed in a cold bath $\left(4: 1(\mathrm{v} / \mathrm{v})\right.$ 2-MeTHF:THF/liq $\left.\mathrm{N}_{2}\left(-145^{\circ} \mathrm{C}\right)\right)$ and oxygenated by bubbling dioxygen via a fine-point needle. The oxygenated sample was maintained at $-145{ }^{\circ} \mathrm{C}$ for $\sim 35$ mins and after generation of $\left[1 \cdot \mathrm{O}_{2}\right] \mathrm{B}\left(\mathrm{C}_{6} \mathrm{~F}_{5}\right)_{4}$, excess TEMPO-H was added to generate TEMPO radical and $[1 \cdot \mathrm{OOH}] \mathrm{B}\left(\mathrm{C}_{6} \mathrm{~F}_{5}\right)_{4}$. Then, the sample tube was frozen in liquid $\mathrm{N}_{2}$. Figure 4 in the main manuscript depicts the EPR data obtained. Generations of $[1 \cdot \mathrm{OOH}] \mathrm{B}\left(\mathrm{C}_{6} \mathrm{~F}_{5}\right)_{4}$ and TEMPO radical are demonstrated by formation of the reverse-axial $\mathrm{Cu}^{\mathrm{II}}$ features and the sharp radical peak respectively. For quantifying the amount of TEMPO radical formed from this reaction, four different solutions $(0.5 \mathrm{mM}, 1.0 \mathrm{mM}, 1.5 \mathrm{mM}, 2.0 \mathrm{mM})$ of pre-sublimed TEMPO radical in 2MeTHF were used as standards and the integrated areas corresponding to the $\mathrm{Cu}^{\mathrm{II}}$-spins were noted. The amount of TEMPO radical in solution is estimated to be $91 \%$. Similarly, the amount of 
$[1 \cdot \mathrm{OOH}] \mathrm{B}\left(\mathrm{C}_{6} \mathrm{~F}_{5}\right)_{4}$ species in solution (when compared with the integrated area of an authentic 1.0 $\mathrm{mM}$ methanolic solution of $\mathrm{Cu}^{\mathrm{II}}$-salt, $[\mathbf{2} \cdot \mathbf{C l}]^{+}$, is estimated to be $97 \%$.

Simulation of the EPR data of $\left[\mathbf{1}^{\cdot} \mathrm{OOH}\right] \mathrm{B}\left(\mathrm{C}_{6} \mathrm{~F}_{5}\right)_{4}$ was challenged by the presence of TEMPO ${ }^{\bullet}$ and by the peak widths. The $g_{z z}$ and $A_{z z}(\mathrm{Cu})$ values could be clearly seen and were fit to be 2.23 and $370 \mathrm{MHz}$, respectively. The $g_{x x}, g_{y y}, A_{x x}(\mathrm{Cu})$, and $A_{y y}(\mathrm{Cu})$ values were obscured. The parameters from fitting routines of EasySpin 5.2.3 were $g_{x x} 2.02, g_{y y} 2.11, A_{x x}(\mathrm{Cu}) 152 \mathrm{MHz}$, and $A_{y y}(\mathrm{Cu})$ $168 \mathrm{MHz}$. The simulated spectrum is overlayed with the data in Figure S15.

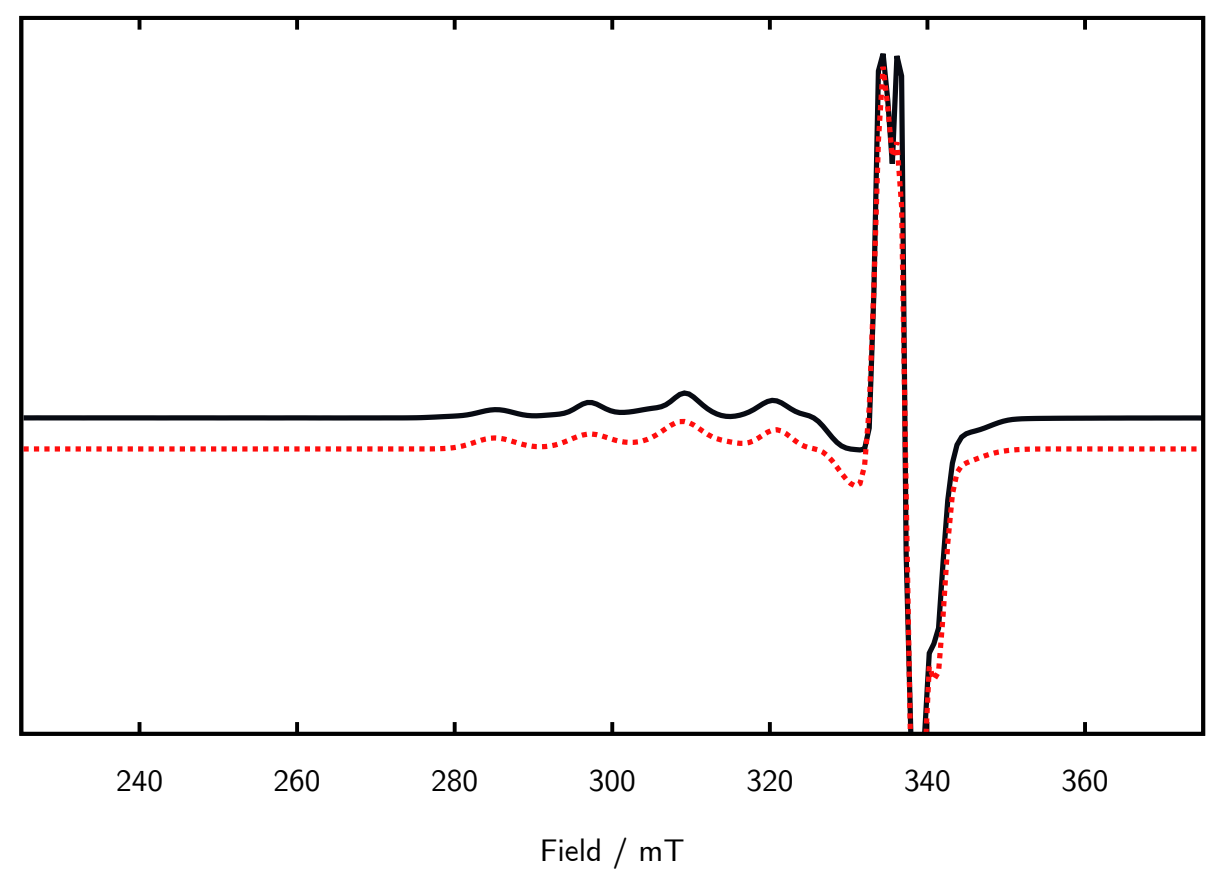

Figure S15. The EPR data could be simulated with EasySpin using best fit copper(II) parameters of $g=[2.2303$, $2.1143,2.0185], \mathrm{A}=[370.3,167.9,152.0]$ for $\mathrm{Nucs}={ }^{\prime} \mathrm{Cu}$ ', $\mathrm{lw}=4.24$, gStrain $=[0.0278,0.0244,0.0407]$. The EasySpin parameters for TEMPO ${ }^{*}$ were $\mathrm{g}=[2.0082,2.0052,2.0022], \mathrm{A}=[16.8,20.4,95.7]$ for Nucs $=$ ' $14 \mathrm{~N}^{\prime}, \mathrm{l} \mathrm{w}=$ 1.85 , gStrain $=[0.0049,0.0090,0.0077]$. 


\section{References}

1. Liang, H. C.; Kim, E.; Incarvito, C. D.; Rheingold, A. L.; Karlin, K. D. A Bis-Acetonitrile Two-Coordinate Copper(I) Complex: Synthesis and Characterization of Highly Soluble $\mathrm{B}\left(\mathrm{C}_{6} \mathrm{~F}_{5}\right)_{4}{ }^{-}$Salts of $\left[\mathrm{Cu}(\mathrm{MeCN})_{2}\right]^{+}$and $\left[\mathrm{Cu}(\mathrm{MeCN})_{4}\right]^{+}$. Inorganic Chemistry 2002, 41, 2209-2212.

2. Würtele, C.; Gaoutchenova, E.; Harms, K.; Holthausen, M. C.; Sundermeyer, J.; Schindler, S. Crystallographic characterization of a synthetic 1:1 end-on copper dioxygen adduct complex. Angewandte Chemie International Edition 2006, 45, 3867-3869.

3. Wittmann, H.; Raab, V.; Schorm, A.; Plackmeyer, J.; Sundermeyer, J. Complexes of Manganese, Iron, Zinc, and Molybdenum with a Superbasic Tris (guanidine) Derivative of Tris (2-ethylamino) amine (Tren) as a Tripod Ligand. European Journal of Inorganic Chemistry 2001, 2001, 1937-1948.

4. Schatz, M.; Raab, V.; Foxon, S. P.; Brehm, G.; Schneider, S.; Reiher, M.; Holthausen, M. C.; Sundermeyer, J.; Schindler, S. Combined Spectroscopic and Theoretical Evidence for a Persistent End-On Copper Superoxo Complex. Angewandte Chemie International Edition 2004, 43, 4360-4363.

5. Maiti, D.; Lee, D. H.; Gaoutchenova, K.; Würtele, C.; Holthausen, M. C.; Narducci Sarjeant, A. A.; Sundermeyer, J.; Schindler, S.; Karlin, K. D. Reactions of a Copper (II) Superoxo Complex Lead to C-H and O-H Substrate Oxygenation: Modeling Copper-Monooxygenase C-H Hydroxylation. Angewandte Chemie International Edition 2008, 47, 82-85.

6. Maiti, D.; Fry, H. C.; Woertink, J. S.; Vance, M. A.; Solomon, E. I.; Karlin, K. D. A 1: 1 Copper-Dioxygen Adduct is an End-on Bound Superoxo Copper (II) Complex which Undergoes Oxygenation Reactions with Phenols. Journal of the American Chemical Society 2007, 129, 264-265.

7. Neuba, A.; Rohrmüller, M.; Hölscher, R.; Schmidt, W. G.; Henkel, G. A panel of peralkylated sulfur-guanidine type bases: Novel pro-ligands for use in biomimetic coordination chemistry. Inorganica Chimica Acta 2015, 430, 225-238.

8. Mader, E. A.; Davidson, E. R.; Mayer, J. M. Large Ground-State Entropy Changes for Hydrogen Atom Transfer Reactions of Iron Complexes. Journal of the American Chemical Society 2007, 129, 5153-5166.

9. Wu, A.; Mader, E. A.; Datta, A.; Hrovat, D. A.; Borden, W. T.; Mayer, J. M. Nitroxyl Radical Plus Hydroxylamine Pseudo Self-Exchange Reactions: Tunneling in Hydrogen Atom Transfer. Journal of the American Chemical Society 2009, 131, $11985-11997$.

10. Fulmer, G. R.; Miller, A. J. M.; Sherden, N. H.; Gottlieb, H. E.; Nudelman, A.; Stoltz, B. M.; Bercaw, J. E.; Goldberg, K. I. NMR Chemical Shifts of Trace Impurities: Common Laboratory Solvents, Organics, and Gases in Deuterated Solvents Relevant to the Organometallic Chemist. Organometallics 2010, 29, 2176-2179.

11. Tenderholt, A.; Hedman, B.; Hodgson, K. O. PySpline: A Modern, Cross-Platform Program for the Processing of Raw Averaged XAS Edge and EXAFS Data. AIP Conference Proceedings 2007, 882, 105-107. 
12. Rehr, J. J.; Albers, R. C. Theoretical approaches to x-ray absorption fine structure. Reviews of Modern Physics 2000, 72 , $621-654$.

13. Neese, F. Software update: the ORCA program system, version 4.0. WIREs Computational Molecular Science 2018, 8, e1327.

14. Neese, F.; Wennmohs, F.; Hansen, A.; Becker, U. Efficient, approximate and parallel Hartree-Fock and hybrid DFT calculations. A 'chain-of-spheres' algorithm for the Hartree-Fock exchange. Chemical Physics 2009, 356, 98.

15. Becke, A. D. Density-functional exchange-energy approximation with correct asymptotic behavior. Physical Review A 1988, 38, 3098-3100.

16. Lee, C.; Yang, W.; Parr, R. G. Development of the Colle-Salvetti correlation-energy formula into a functional of the electron density. Physical Review B 1988, 37, 785-789.

17. Grimme, S.; Ehrlich, S.; Goerigk, L. Effect of the damping function in dispersion corrected density functional theory. Journal of Computational Chemistry 2011, 32, 1456-1465.

18. Grimme, S.; Antony, J.; Ehrlich, S.; Krieg, H. A consistent and accurate ab initio parametrization of density functional dispersion correction (DFT-D) for the 94 elements H-Pu. The Journal of Chemical Physics 2010, 132, 154104.

19. Weigend, F.; Ahlrichs, R. Balanced basis sets of split valence, triple zeta valence and quadruple zeta valence quality for H to Rn: Design and assessment of accuracy. Physical Chemistry Chemical Physics 2005, 7, 3297-3305.

20. Weigend, F. Accurate Coulomb-fitting basis sets for H to Rn. Physical Chemistry Chemical Physics 2006, 8, $1057-1065$.

21. Barone, V.; Cossi, M. Quantum Calculation of Molecular Energies and Energy Gradients in Solution by a Conductor Solvent Model. The Journal of Physical Chemistry A 1998, 102, 1995-2001.

22. Bhadra, M.; Lee, J. Y. C.; Cowley, R. E.; Kim, S.; Siegler, M. A.; Solomon, E. I.; Karlin, K. D. Intramolecular hydrogen bonding enhances stability and reactivity of mononuclear cupric superoxide complexes. Journal of the American Chemical Society 2018, 140, 9042-9045. 